\title{
BOUNDARY CONTROLLABILITY OF THE FINITE-DIFFERENCE SPACE SEMI-DISCRETIZATIONS OF THE BEAM EQUATION*,**
}

\author{
LILIANA LEÓN ${ }^{1}$ AND ENRIQUE ZUAZUA ${ }^{2}$
}

\begin{abstract}
We propose a finite difference semi-discrete scheme for the approximation of the boundary exact controllability problem of the 1-D beam equation modelling the transversal vibrations of a beam with fixed ends. First of all we show that, due to the high frequency spurious oscillations, the uniform (with respect to the mesh-size) controllability property of the semi-discrete model fails in the natural functional setting. We then prove that there are two ways of restoring the uniform controllability property: a) filtering the high frequencies, i.e. controlling projections on subspaces where the high frequencies have been filtered; $b$ ) adding an extra boundary control to kill the spurious high frequency oscillations. In both cases the convergence of controls and controlled solutions is proved in weak and strong topologies, under suitable assumptions on the convergence of the initial data.
\end{abstract}

Mathematics Subject Classification. 93C20, 35Q33, 65N06.

Received November 2, 2001.

\section{INTRODUCTION}

The transversal vibrations of a 1-d beam with hinged boundary conditions are modelled by the following equation

$$
\begin{cases}u^{\prime \prime}+\partial_{x}^{4} u=0 & \text { in } Q=(0,1) \times(0, T) \\ u(0, t)=u(1, t)=0 & t \in(0, T) \\ \partial_{x}^{2} u(0, t)=\partial_{x}^{2} u(1, t)=0 & t \in(0, T) \\ u(x, 0)=u^{0}(x), \quad u^{\prime}(x, 0)=u^{1}(x) & x \in(0,1),\end{cases}
$$

where ' denotes the derivative of $u$ with respect to time.

Keywords and phrases: Beam equation, finite difference semi-discretization, exact boundary controllability.

* This work was done while the first author was visiting Universidad Complutense de Madrid with a fellowship of the Alpha Program of the European Union "Modélisation 8 Ingénierie Mathématique".

** Partially supported by grant PB96-0663 of the DGES (Spain) and the TMR network of the EU "Homogenization Es Multiple Scales".

${ }^{1}$ Laboratório de Ciências Matemáticas, Universidade Estadual do Norte Fluminense, 28015 Rio de Janeiro, Brazil; e-mail: lalm@uenf.br

2 Departamento de Matemáticas, Facultad de Ciencias, Universidad Autónoma, Cantoblanco, 28049 Madrid, Spain; e-mail: enrique.zuazua@uam.es 
When $\left(u^{0}, u^{1}\right) \in H_{0}^{1}(0,1) \times H^{-1}(0,1)$ there exists a unique solution $u$ such that

$$
u \in C\left([0, T] ; H_{0}^{1}(0,1)\right) \cap C\left([0, T] ; H^{-1}(0,1)\right) .
$$

This solution admits the Fourier expansion

$$
u(x, t)=\sum_{k=1}^{\infty}\left\{a_{k} \cos k^{2} \pi^{2} t+b_{k} \sin k^{2} \pi^{2} t\right\} \sin k \pi x,
$$

with suitable Fourier coefficients depending on the initial data $\left(u^{0}, u^{1}\right)$.

The energy associated with $\left(u^{0}, u^{1}\right) \in H_{0}^{1}(0,1) \times H^{-1}(0,1)$ is given by

$$
E(t)=\frac{1}{2}\left\{\|u(., t)\|_{1}^{2}+\left\|u^{\prime}(., t)\right\|_{-1}^{2}\right\},
$$

where $\|\cdot\|_{1}$ and $\|\cdot\|_{-1}$ are the canonical norms in $H_{0}^{1}(0,1)$ and $H^{-1}(0,1)$, respectively. Namely

$$
\|u\|_{1}=\left[\int_{0}^{1}\left(\partial_{x} u\right)^{2} \mathrm{~d} x\right]^{1 / 2} ; \quad\|u\|_{-1}=\left\|\left(-\partial_{x}^{2}\right)^{-1} u\right\|_{1}
$$

where $\left(-\partial_{x}^{2}\right)^{-1}$ denotes the inverse of the operator $-\partial_{x}^{2}$ with homogeneous Dirichlet boundary conditions at $x=0,1$.

It is easy to see that the energy $E(t)$ is conserved along time for the solutions of (1).

Applying multipliers or Fourier series techniques one can prove a boundary observability inequality showing that, for every $T>0$, there exists $C=C(T)>0$ such that

$$
E(0) \leq C \int_{0}^{T}\left|\partial_{x} u(1, t)\right|^{2} \mathrm{~d} t
$$

for every solution of (1) (see Lions [10]).

As a consequence of this observability inequality and Lions' HUM method [10] the following boundary controllability property may be proved:

For all $T>0$ and $\left(y^{0}, y^{1}\right) \in F=H_{0}^{1}(0,1) \times H^{-1}(0,1)$, there exists a control $\nu \in L^{2}(0, T)$, such that the solution of

$$
\begin{cases}y^{\prime \prime}+\partial_{x}^{4} y=0 & \text { in } Q=I \times(0, T) \\ y(0, t)=y(1, t)=0 & t \in(0, T) \\ \partial_{x}^{2} y(0, t)=0 \quad \partial_{x}^{2} y(1, t)=\nu & t \in(0, T) \\ y(x, 0)=y^{0}(x), \quad y^{\prime}(x, 0)=y^{1}(x) & x \in I,\end{cases}
$$

satisfies

$$
y(x, T, \nu)=y^{\prime}(x, T, \nu)=0 .
$$

The main objective of this work is to study the controllability of the classical semi-discrete space approximation by finite differences of (6). We also study the convergence of controls and controlled solutions as the mesh-size tends to zero. Our work provides two alternative methods for the numerical approximation of the exact control $\nu$ of equation (6). 
In Section 2 we analyze the problem of the observability of the finite-difference space semi-discretization of the beam equation (1). In Section 3, combining the results of the previous section, we find two results of uniform controllability (as the mesh size tends to zero) of the semi-discrete space approximation by finite differences of (6). The first one concerns the partial control obtained by filtering the high frequencies and the second one the control of the semi-discrete solutions by means of a suitable modification of the boundary control. In Section 4, we study the convergence of the solutions of the previous semi-discrete problems as the mesh-size tends to zero.

\section{ANALYSis OF THE BOUNDARY OBSERVABILITY PROBLEM}

For each $N \in \mathbb{N}$ we consider a partition of $I=(0,1), \mathcal{P}=\left\{x_{0}=0, \ldots, x_{j}=j h, \ldots, x_{N+1}=1\right\}$, where the mesh-size is $h=1 /(N+1)$.

To get a discrete definition of the boundary conditions of the problem (1) using centered finite differences, we also introduce two external points $x_{-1}=x_{0}-h$ and $x_{N+2}=x_{N+1}+h$. We denote by $u_{h, j}(t)$ the approximation of the solution of (1) at the point $x_{j}$. We also set $u_{h,-1}=-u_{h, 1}$ and $u_{h, N+2}=-u_{h, N}$.

The semi-discretization by finite differences of (1) is then given by the following system of $N$ ordinary differential equations,

$$
\left\{\begin{array}{l}
u_{h, j}^{\prime \prime}=-\frac{1}{h^{4}}\left[u_{h, j+2}-4 u_{h, j+1}+6 u_{h, j}-4 u_{h, j-1}+u_{h, j-2}\right] \\
0<t<T \quad j=1,2, \ldots N \\
u_{h, 0}=u_{h, N+1}=0, \quad 0<t<T \\
u_{h,-1}=-u_{h, 1} \quad u_{h, N+2}=-u_{h, N}, \quad 0<t<T \\
u_{h, j}(0)=u_{h, j}^{0} \quad u_{h, j}^{\prime}(0)=u_{h, j}^{1}, j=1,2, \ldots N .
\end{array}\right.
$$

Here the initial conditions $\left(u_{j}^{0}, u_{j}^{1}\right)$ of $(7)$ are suitable approximations of the initial conditions of $(1)$ at the points $x_{j}$ of the mesh. It is easy to see that the scheme $(7)$ is convergent as $h \rightarrow 0$ in the classical sense, i.e. it is consistent and stable.

The eigenvalue problem associated to (7) is as follows

$$
\left\{\begin{array}{l}
\frac{1}{h^{4}} B \vec{\phi}^{k}(h)=\beta \vec{\phi}^{k}(h) \\
\phi_{k, 0}=\phi_{k, N+1} \\
\phi_{k,-1}=-\phi_{k, 1} \quad \phi_{k, N+2}=-\phi_{k, N}
\end{array}\right.
$$

where

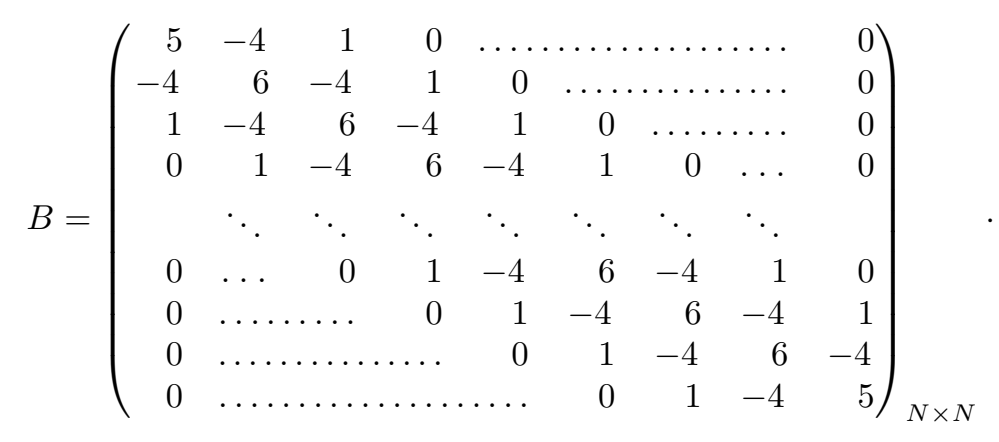




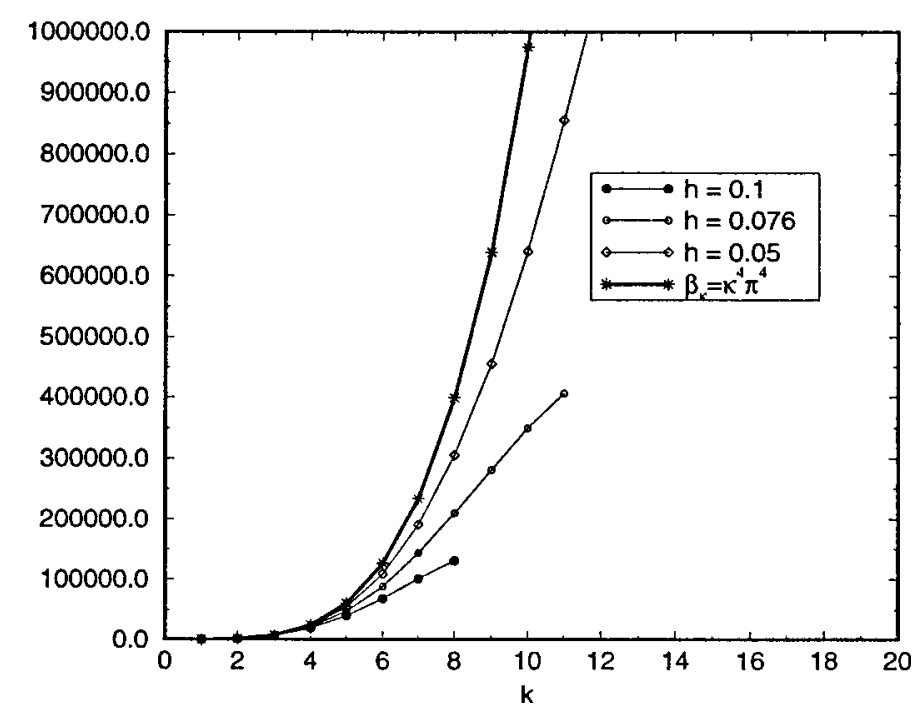

FiguRE 1. Graph of discrete eigenvalues $\beta_{k}(h)$ for different values of $h$ and the continuous eigenvalues $\beta_{k}=k^{4} \pi^{4}$ of the beam equation.

Note that $B=A^{2}$ with

$$
A=\left(\begin{array}{rrrrrrr}
-2 & 1 & 0 & \ldots \ldots \ldots \ldots & 0 \\
1 & -2 & 1 & 0 & \ldots \ldots \ldots & 0 \\
0 & 1 & -2 & 1 & 0 & \ldots & 0 \\
& \ddots & \ddots & \ddots & \ddots & \ddots & \\
0 & \ldots & 0 & 1 & -2 & 1 & 0 \\
0 & \ldots & \ldots & 0 & 1 & -2 & 1 \\
0 & \ldots & \ldots & \ldots & 0 & 1 & -2
\end{array}\right)_{N \times N}
$$

The matrix $A$ arises in the semi-discrete approximation of Laplace's equation in one space dimension and its eigenvalues and eigenvectors are well known (see [6]);

$$
\begin{aligned}
& \lambda_{k}(h)=\frac{4}{h^{2}} \sin ^{2}\left(\frac{k \pi h}{2}\right) \quad k=1,2, \ldots, N \\
& \vec{\phi}^{k}(h)=\left(\phi_{k, 1}, \phi_{k, 2}, \ldots, \phi_{k, N}\right) \quad k=1,2, \ldots, N \\
& \phi_{k, j}(h)=\sin (j k \pi h) .
\end{aligned}
$$

Then, the eigenvectors of $B$ are the same, and their eigenvalues are

$$
\beta_{k}(h)=\lambda_{k}^{2}(h)=\frac{16}{h^{4}} \sin ^{4}\left(\frac{k \pi h}{2}\right) \quad k=1,2, \ldots, N
$$

The discrete eigenvalues $\beta_{k}(h)$ approximate the eigenvalues of the continuous model $\beta_{k}=k^{4} \pi^{4}$ for $k$ fixed, when the size of the mesh $h$ tends to zero, and its eigenvectors $\vec{\phi}^{k}(h)$ coincide with the eigenfunctions $\phi^{k}(x)=\sin (k \pi x)$ of the continuous model (1) on the mesh points. 
Therefore, the solution of system (7) may be expressed as

$$
\vec{u}_{h}(t)=\sum_{k=1}^{N}\left\{a_{k} \cos \left(\sqrt{\beta_{k}(h)} t\right)+b_{k} \sin \left(\sqrt{\beta_{k}(h)} t\right)\right\} \vec{\phi}^{k}(h)
$$

with $\vec{u}_{h}(t)=\left(u_{h, 1}(t), u_{h, 2}(t), \ldots u_{h, N}(t)\right)$, for suitable Fourier coefficients $a_{j}, b_{j}$ depending on the initial data.

We set $A_{h}=\frac{1}{h^{2}} A$, where $A$ is as in (10) and we denote by $A_{h}^{-1}$ its inverse. We define the energy $E^{h}(t)$ associated to problem (7) by

$$
E^{h}(t)=\frac{h}{2} \sum_{j=1}^{N}\left\{\left|\frac{u_{h, j+1}(t)-u_{h, j}(t)}{h}\right|^{2}+\left|\frac{\left(A_{h}^{-1}{\overrightarrow{u_{h}}}^{\prime}(t)\right)_{j+1}-\left(A_{h}^{-1}{\overrightarrow{u_{h}}}^{\prime}(t)\right)_{j}}{h}\right|^{2}\right\}
$$

which is an approximation of the continuous energy $E(t)$. Note that $E_{h}(t)$ is conserved along time for solutions of $(7)$.

It is then natural to analyze the following semi-discrete version of the observability inequality (5):

$$
E^{h}(0) \leq C(T, h) \int_{0}^{T}\left|\frac{u_{h, N}(t)}{h}\right|^{2} \mathrm{~d} t
$$

where $C(T, h)$ is independent of the solution of $(7)$.

The observability inequality (16) is said to be uniform, if the constants $C(T, h)$ are bounded uniformly in $h$, as $h \rightarrow 0$.

However, as we shall see below in Section 2.1, whatever $T>0$ is, the inequality (16) may not be uniform.

In order to restore the uniformity of the observability inequality with respect to $h$ there are two possibilities: (a) to restrict the class of solutions of (7) under consideration; (b) to reinforce the observed quantity on the right hand side of (16).

Once the lack of uniform observability is proved in Section 2.1 the rest of this section will be devoted to prove the two uniform observability properties mentioned above.

\subsection{Non-uniform observability}

Let us first recall the following observability identity for the eigenvectors of $B$ (see Lem. 1.1 in [5])

$$
h \sum_{j=0}^{N}\left|\frac{\phi_{k, j+1}-\phi_{k, j}}{h}\right|^{2}=\frac{2}{\left(4-\sqrt{\beta_{k}(h)} h^{2}\right)}\left|\frac{\phi_{k, N}}{h}\right|^{2} \quad k=1,2, \ldots N .
$$

This identity allows to show that the observability inequality may not be uniform as $h \rightarrow 0$ for any $T$. More precisely, we have the following negative result:

Theorem 2.1. For any $T>0$, we have

$$
\sup _{\vec{u}_{h} \text { sol. of (7) }}\left(\frac{E^{h}(0)}{\int_{0}^{T}\left|u_{h, N}(t) / h\right|^{2} \mathrm{~d} t}\right) \longrightarrow \infty, \quad \text { as } \quad h \rightarrow 0 .
$$

Proof. For $h>0$, consider $\vec{u}_{h}(t)=\left(u_{h, 1}(t), u_{h, 2}(t), \ldots, u_{h, N}(t)\right)$ the solution of $(7)$, associated to the eigenfunction $\vec{\phi}^{k}(h)$ :

$$
\vec{u}_{h}(t)=\mathrm{e}^{i \sqrt{\beta_{k}(h)} t} \vec{\phi}^{k}(h)=\mathrm{e}^{i \lambda_{k}(h) t} \vec{\phi}^{k}(h) .
$$


According to (17)

$$
\int_{0}^{T}\left|\frac{u_{h, N}(t)}{h}\right|^{2} \mathrm{~d} t=\left|\frac{\phi_{k, N}}{h}\right|^{2} \int_{0}^{T}\left|\mathrm{e}^{i \lambda_{k}(h) t}\right|^{2} \mathrm{~d} t=\frac{T}{2}\left(4-\lambda_{k}(h) h^{2}\right) h \sum_{j=0}^{N}\left|\frac{\phi_{k, j+1}-\phi_{k, j}}{h}\right|^{2} .
$$

Note that $4-\lambda_{k}(h) h^{2}=4 \cos ^{2}\left(\frac{k \pi h}{2}\right)$.

On the other hand,

$$
E^{h}(0)=h \sum_{j=0}^{N}\left|\frac{\phi_{k, j+1}-\phi_{k, j}}{h}\right|^{2}
$$

Therefore

$$
E^{h}(0)=\frac{1}{2 T \cos ^{2}\left(\frac{k \pi h}{2}\right)} \int_{0}^{T}\left|\frac{u_{h, N}(t)}{h}\right|^{2} \mathrm{~d} t
$$

Thus for any $T>0$, taking $k=N$ and using that

$$
\cos ^{2}\left(\frac{N \pi h}{2}\right)=\cos ^{2}\left(\frac{\pi}{2}-\frac{\pi h}{2}\right) \longrightarrow 0 \quad \text { when } \quad h \rightarrow 0,
$$

equation (18) holds immediately.

Remark 2.1. Let $\delta \in(0,1)$ be given. The counterexample above may not be found in the class of low frequency solutions with Fourier components corresponding to indexes $k \leq \delta N$. Indeed in that case, the quotient in (21) may be bounded below by $1 /\left[2 T \cos ^{2} \delta((\pi-\pi h) / 2)\right]$ which is bounded as $h \longrightarrow 0$.

This observation motivates the uniform observability result we state in the following section for filtered solutions in which the high frequency components have been filtered.

\subsection{Uniform observability of filtered solutions}

Given $\gamma \in(0,16)$ and $h>0$, we consider $\mathbf{C}_{h}(\gamma)$ the class of solutions of (7) generated by the eigenvectors of (8) associated with eigenvalues such that

$$
\beta_{k}(h) \leq \gamma h^{-4}
$$

More precisely,

$$
\mathbf{C}_{h}(\gamma)=\left\{\vec{u}_{h} \quad \text { sol. of }(7): \vec{u}_{h}=\sum_{\beta_{k}(h) \leq \gamma h^{-4}}\left\{a_{k} \mathrm{e}^{i \sqrt{\beta_{k}(h)} t}+b_{k} \mathrm{e}^{-i \sqrt{\beta_{k}(h)} t}\right\} \vec{\phi}^{k}(h)\right\}
$$

Observe that, when $\gamma=16, \mathbf{C}_{h}(\gamma)=\mathbf{C}_{h}(16)$ coincides with the space of all solutions of the semi-discrete problem (7). The following observability result holds in this class.

Theorem 2.2. Let $0<\gamma<16$. For all $T>0$, there exists $C=C(T, \gamma)>0$ such that

$$
E^{h}(0) \leq C \int_{0}^{T}\left|\frac{u_{h, N}(t)}{h}\right|^{2} \mathrm{~d} t \quad \forall \vec{u}_{h} \in \mathbf{C}_{h}(\gamma), \forall h>0
$$

The proof of this result relies on Ingham's inequality (see [17] for instance) in which the gap between the consecutive eigenvalues of the semi-discrete system (7) plays a crucial role. 


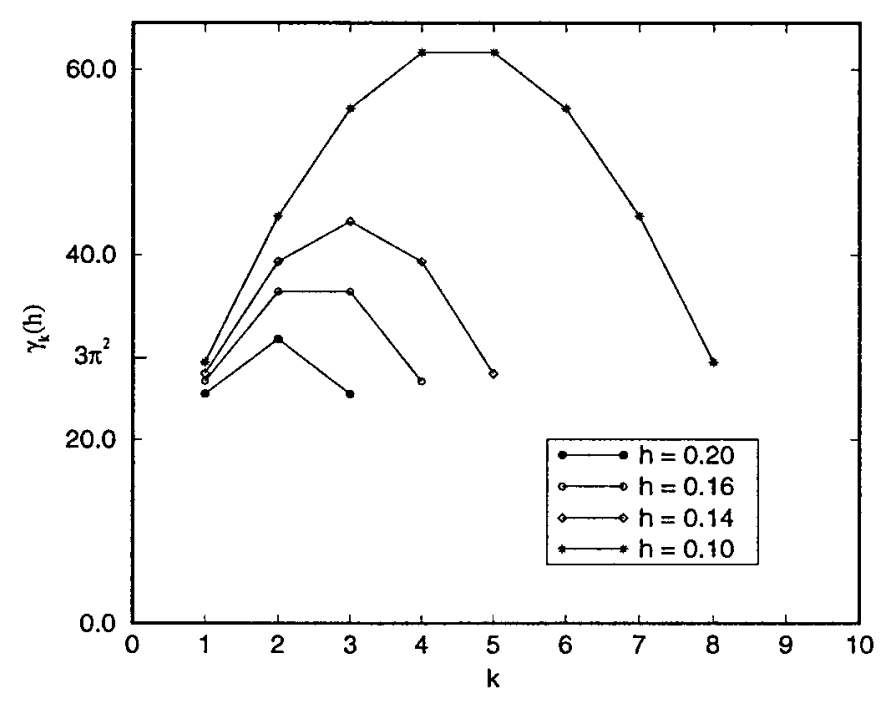

FIGURE 2. The function $k \rightarrow \gamma_{k}(h)$ for different values of $h$.

\subsubsection{Analysis of the gap between consecutive eigenvalues}

Let us first observe that the gap for the continuous model (1) satisfies

$$
\sqrt{\beta_{k+1}}-\sqrt{\beta_{k}}=(2 k+1) \pi^{2} \rightarrow \infty
$$

Moreover, we have the following:

Lemma 2.1. The following properties hold:

$$
\begin{aligned}
& \text { (i) } \sqrt{\beta_{k+1}(h)}-\sqrt{\beta_{k}(h)} \geq 3 \pi^{2}\left\{\frac{\sin \frac{\pi h}{2}}{\frac{\pi h}{2}}\right\}^{2}-\pi^{4} h^{2} \\
& \text { (ii) } \lim _{h \rightarrow 0} \inf _{1 \leq k \leq \frac{1}{h}-2}\left\{\sqrt{\beta_{k+1}(h)}-\sqrt{\beta_{k}(h)}\right\}=3 \pi^{2} \\
& \text { (iii) } \lim _{h \rightarrow 0} \sup _{1 \leq k \leq \frac{1}{h}-2}\left\{\sqrt{\beta_{k+1}(h)}-\sqrt{\beta_{k}(h)}\right\}=\infty .
\end{aligned}
$$

Proof. (i) We set

$$
\gamma_{k}(h)=\sqrt{\beta_{k+1}(h)}-\sqrt{\beta_{k}(h)}, \quad \text { for } \quad k=1,2, \ldots, N-1
$$

Using classical trigonometrical identities it follows that

$$
\gamma_{k}(h)=\frac{2}{h^{2}}(\cos k \pi h-\cos (k+1) \pi h)=\frac{4}{h^{2}} \sin \frac{(2 k+1) \pi h}{2} \sin \frac{\pi h}{2} .
$$

We observe that $\quad 0<\frac{3 \pi h}{2} \leq \frac{(2 k+1) \pi h}{2} \leq \pi-\frac{3 \pi h}{2} \quad$ for $k=1,2 \ldots, N-1$.

Thus, for every $h>0$, the function $k \rightarrow \gamma_{k}(h)$ describes a concave parabola which is symmetric with respect to $k=\frac{1-h}{2 h}$, as shown in Figure 2 . 
Then, it is enough to consider $k \in \mathbb{N}$ such that $\frac{3 \pi h}{2} \leq \frac{(2 k+1) \pi h}{2} \leq \frac{\pi}{2}$, or equivalently $1 \leq k \leq \frac{1-h}{2 h}$. For those indexes $k$ we have

$$
\gamma_{k}(h) \geq \frac{4}{h^{2}} \sin \left\{\frac{3 \pi h}{2}\right\} \sin \left\{\frac{\pi h}{2}\right\}, \quad \forall k \in \mathbb{N} \cap\left[1, \frac{1-h}{2 h}\right] .
$$

Using the trigonometrical identity $\sin 3 \alpha=3 \sin \alpha-4 \sin ^{3} \alpha \quad$ in the previous inequality, we conclude that

$$
\gamma_{k}(h) \geq 3 \pi^{2}\left\{\frac{\sin \frac{\pi h}{2}}{\frac{\pi h}{2}}\right\}^{2}-\pi^{4} h^{2}\left\{\frac{\sin \frac{\pi h}{2}}{\frac{\pi h}{2}}\right\}^{4} \geq 3 \pi^{2}\left\{\frac{\sin \frac{\pi h}{2}}{\frac{\pi h}{2}}\right\}^{2}-\pi^{4} h^{2},
$$

for every $k=1,2, \ldots, N-1$.

(ii) From (27) we have that

$$
\inf _{1 \leq k \leq \frac{1}{h}-2}\left\{\gamma_{k}(h)\right\}=\inf _{1 \leq k \leq \frac{1}{h}-2}\left\{\frac{4}{h^{2}} \sin \frac{(2 k+1) \pi h}{2} \sin \frac{\pi h}{2}\right\}=\frac{4}{h^{2}} \sin \frac{3 \pi h}{2} \sin \frac{\pi h}{2} .
$$

Then, (25) holds.

(iii) Again from the equality (27) we have that

$$
\sup _{1 \leq k \leq \frac{1}{h}-2}\left\{\gamma_{k}(h)\right\}=\sup _{1 \leq k \leq \frac{1}{h}-2}\left\{\frac{4}{h^{2}} \sin \frac{(2 k+1) \pi h}{2} \sin \frac{\pi h}{2}\right\}=\frac{4}{h^{2}} \sin \frac{\pi}{2} \sin \frac{\pi h}{2},
$$

and (26) holds.

We also have the following additional property:

Lemma 2.2. For all $\gamma_{\infty}>0$, there exist $\delta>0$ and $k_{0} \in \mathbb{N}$ such that:

$$
\sqrt{\beta_{k+1}(h)}-\sqrt{\beta_{k}(h)} \geq \gamma_{\infty}
$$

for $k=k_{0}, k_{0}+1, \ldots, 1 / h-1-k_{0}$ and $\forall|h|<\delta$.

Proof. In view of (29) there exists $k_{0}$ and $\delta>0$ such that

$$
\gamma_{k_{0}}(h) \geq \gamma_{\infty} \quad \text { for all }|h|<\delta
$$

Then, taking into account that the parabola $k \longrightarrow \gamma_{k}(h)$ is symmetric with respect to $k=\frac{1-h}{2 h}$, we deduce that

$$
\gamma_{k}(h) \geq \gamma_{\infty} \quad \text { for } \quad k=k_{0}, \ldots, \frac{1-2 h}{h}-k_{0}
$$

In view of the particular structure of the gap functions described above we need the following variant of Ingham's inequality, whose proof is very close to that of Theorem 3.4 given in [13].

Lemma 2.3. Let $f(t)=\sum_{n \in \mathbb{Z}} d_{n} \mathrm{e}^{i \mu_{n} t}$ where $\left\{\mu_{n}\right\}_{n \in \mathbb{Z}}$ is a sequence of real numbers, such that there exist $N \in \mathbb{N}, \gamma>0$ and $\gamma_{\infty}>0$ such that

(i) $\quad \mu_{n+1}-\mu_{n} \geq \gamma \quad \forall n \in \mathbb{Z}$.

(ii) $\operatorname{Card}\left\{n \in \mathbb{Z}: \mu_{n+1}-\mu_{n} \leq \gamma_{\infty}\right\}=N$. 
Then, for any interval $J=[0, T] \subset \mathbb{R}$ with $T>\frac{2 \pi}{\gamma_{\infty}}$ there exist two positive constants $C_{1}, C_{2}>0$ such that

$$
C_{1} \sum_{n \in \mathbb{Z}}\left|d_{n}\right|^{2} \leq \int_{0}^{T}\left|\sum_{n \in \mathbb{Z}} d_{n} \mathrm{e}^{i \mu_{n} t}\right|^{2} \mathrm{~d} t \leq C_{2} \sum_{n \in \mathbb{Z}}\left|d_{n}\right|^{2}
$$

for all sequence $\left\{d_{n}\right\} \in l^{2}$.

More precisely $C_{1}=C_{1}(N)$ and $C_{2}=C_{2}(N)$, where $C_{i}(j), i=1,2$, are given by the following recurrent formulas

$$
\begin{aligned}
C_{1}(j+1) & =\left[\left(2 \frac{C_{2}(j)}{T}+1\right) \frac{4}{C_{1}(j)\left(T \gamma_{\infty}-2 \pi\right)^{2} \gamma^{2}}+\frac{2}{T}\right]^{-1}, \\
C_{2}(j) & =2\left\{j T+C_{2}(0)\right\}, \quad j=1,2, \ldots,
\end{aligned}
$$

and $C_{1}(0), C_{2}(0)$ are such that (33) holds in the particular case in which $N=0$.

Remark 2.2. The main difference between Lemma 2.3 and that proved in [13] is that, here, the set of badly separated $\mu_{n}$-s is not necessarily constituted by the first $n$-s such that $|n| \leq k_{0}$ for some finite $k_{0}$ as in [13].

Proof. We proceed as in [3] and [13]. The proof is divided in two steps.

Step 1. We fix any $T>2 \pi / \gamma_{\infty}$. Let us consider the set

$$
Y=\left\{n \in \mathbb{Z}: \mu_{n+1}-\mu_{n} \leq \gamma_{\infty}\right\}
$$

Denote

$$
g(t)=\sum_{\substack{n \in \mathbb{Z} \\ n \notin Y}} d_{n} \mathrm{e}^{i \mu_{n} t}
$$

Applying Ingham's inequality [13] to $g(t)$, we have that there exist two constants $C_{1}(0)>0$ and $C_{2}(0)>0$ such that:

$$
C_{1}(0) \sum_{\substack{n \in \mathbb{Z} \\ n \notin Y}}\left|d_{n}\right|^{2} \leq \int_{0}^{T}|g(t)|^{2} \mathrm{~d} t \leq C_{2}(0) \sum_{\substack{n \in \mathbb{Z} \\ n \notin Y}}\left|d_{n}\right|^{2} .
$$

For

$$
f(t)=g(t)+\sum_{\substack{n \in \mathbb{Z} \\ n \in Y}} d_{n} \mathrm{e}^{i \mu_{n} t}
$$

we have

$$
\int_{0}^{T}|f(t)|^{2} \mathrm{~d} t=\int_{0}^{T}\left|\sum_{n \in Y} d_{n} \mathrm{e}^{i \mu_{n} t}+\sum_{n \notin Y} d_{n} \mathrm{e}^{i \mu_{n} t}\right|^{2} \leq 2 \int_{0}^{T}\left(\left|\sum_{n \in Y} d_{n} \mathrm{e}^{i \mu_{n} t}\right|^{2}+\left|\sum_{n \notin Y} d_{n} \mathrm{e}^{i \mu_{n} t}\right|^{2}\right) \mathrm{d} t
$$


According to (35) we obtain

$$
\begin{aligned}
\int_{0}^{T}|f(t)|^{2} \mathrm{~d} t & \leq 2 C_{2}(0) \sum_{n \notin Y}\left|d_{n}\right|^{2}+2 T\left(\sum_{n \in Y}\left|d_{n}\right|\right)^{2} \\
& \leq 2 C_{2}(0) \sum_{n \notin Y}\left|d_{n}\right|^{2}+2 T N \sum_{n \in Y}\left|d_{n}\right|^{2} \leq\left(2 C_{2}(0)+2 T N\right) \sum_{n \in \mathbb{Z}}\left|d_{n}\right|^{2} .
\end{aligned}
$$

This provides the second inequality in (33).

Step 2. We now argue by induction on $N$.

If $N=0$, equation (33) follows from (35). When $N=1$, if $Y=\left\{d_{n_{1}}\right\}$, then

$$
f(t)=\sum_{\substack{n \in \mathbb{Z} \\ n \notin Y}} d_{n} \mathrm{e}^{i \mu_{n} t}+d_{n_{1}} \mathrm{e}^{i \mu_{n_{1}} t}
$$

In Theorem 3.4 of [13], it was proved that for $\eta>0$ and $T^{\prime}=T-\eta$, when $f(t)=g(t)+d_{n_{1}}$, then

$$
\gamma^{2} \eta^{4} C_{1}(0) \sum_{\substack{n \in \mathbb{Z} \\ n \notin Y}}\left|d_{n}\right|^{2} \leq \int_{0}^{T^{\prime}}\left|\int_{0}^{\eta}(f(t+\psi)-f(t)) \mathrm{d} \psi\right|^{2} \mathrm{~d} t \leq 4 \eta^{2} \int_{0}^{T}|f(t)|^{2} \mathrm{~d} t .
$$

On the other hand,

$$
\begin{aligned}
\left|d_{n_{1}}\right|^{2} & \leq|f(t)-g(t)|^{2}=\frac{1}{T} \int_{0}^{T}|f(t)-g(t)|^{2} \mathrm{~d} t \leq \frac{2}{T}\left\{\int_{0}^{T}|f(t)|^{2} \mathrm{~d} t+\int_{0}^{T}|g(t)|^{2} \mathrm{~d} t\right\} \\
& \leq \frac{2}{T}\left\{\int_{0}^{T}|f(t)|^{2} \mathrm{~d} t+C_{2}(0) \sum_{\substack{n \in \mathbb{Z} \\
n \notin Y}}\left|d_{n}\right|^{2}\right\} \leq\left\{\frac{2}{T}+\frac{8 C_{2}(0)}{T \gamma^{2} \eta^{2} C_{1}(0)}\right\} \int_{0}^{T}|f(t)|^{2} \mathrm{~d} t .
\end{aligned}
$$

Then, from (38) and (39) we have

$$
\begin{aligned}
\sum_{n \in \mathbb{Z}}\left|d_{n}\right|^{2} & \leq\left\{\frac{4}{\gamma \eta^{2} C_{1}(0)}+\frac{2}{T}+\frac{8 C_{2}(0)}{T \gamma^{2} \eta^{2} C_{1}(0)}\right\} \int_{0}^{T}|f(t)|^{2} \mathrm{~d} t \\
& \leq\left\{\frac{2}{T}+\frac{4}{\gamma^{2} \eta^{2} C_{1}(0)}\left(\frac{2 C_{2}(0)}{T}+1\right)\right\} \int_{0}^{T}|f(t)|^{2} \mathrm{~d} t
\end{aligned}
$$

If $N=2$, let $Y=\left\{n_{1}, n_{2}\right\}$. In particular, if $n_{1}=0$, we write $f(t)$ as

$$
f(t)=\sum_{\substack{n \in \mathbb{Z} \\ n \notin Y}} d_{n} \mathrm{e}^{i \mu_{n} t}+d_{0} \mathrm{e}^{i \mu_{n_{1}} t}+d_{n_{2}} \mathrm{e}^{i \mu_{n_{2}} t} .
$$

Setting

$$
g(t)=\sum_{\substack{n \in \mathbb{Z} \\ n \neq n_{2}}} d_{n} \mathrm{e}^{i \mu_{n} t}+d_{0}
$$


and applying the result in step 1 to $g(t)$ we have

$$
\sum_{\substack{n \in \mathbb{Z} \\ n \neq n_{2}}}\left|d_{n}\right|^{2} \leq\left\{\frac{2}{T}+\frac{4}{\gamma^{2} \eta^{2} C_{1}(0)}\left(\frac{2 C_{2}(0)}{T}+1\right)\right\} \int_{0}^{T}|g(t)|^{2} \mathrm{~d} t
$$

and

$$
\left(2 C_{2}(0)+2 T\right) \sum_{\substack{n \in \mathbb{Z} \\ n \neq n_{2}}}\left|d_{n}\right|^{2} \geq \int_{0}^{T}|g(t)|^{2} \mathrm{~d} t
$$

Thus, from the previous estimate it follows that

$$
\begin{aligned}
\left|d_{n_{2}}\right|^{2} & \leq|f(t)-g(t)|^{2}=\frac{1}{T} \int_{0}^{T}|f(t)-g(t)|^{2} \mathrm{~d} t \leq \frac{2}{T}\left\{\int_{0}^{T}|f(t)|^{2} \mathrm{~d} t+\int_{0}^{T}|g(t)|^{2} \mathrm{~d} t\right\} \\
& \leq \frac{2}{T}\left\{\int_{0}^{T}|f(t)|^{2} \mathrm{~d} t+\left(2 C_{2}(0)+2 T\right) \sum_{k \in \mathbb{Z}-\left\{n_{2}\right\}}\left|d_{n}\right|^{2}\right\} \\
& \leq\left\{\frac{2}{T}+\frac{4}{\gamma^{2} \eta^{2} C_{1}(0)}\left(\frac{4 C_{2}(0)}{T}+4\right)\right\} \int_{0}^{T}|f(t)|^{2} \mathrm{~d} t
\end{aligned}
$$

Iterating this argument the first inequality in (33) follows.

\subsubsection{Proof of Theorem 2.2}

In order to represent the solution in $\mathbf{C}_{h}(\gamma)$ in a simpler way we introduce

$$
I_{h}(\gamma)=\left\{k \in \mathbb{N}: 1 \leq k<\frac{1}{h}-2 \text { and } \beta_{k}(h) \leq \gamma h^{-4}\right\}
$$

and the functions

$$
\begin{gathered}
m_{k, j}= \begin{cases}c_{k}^{+} \phi_{k, j} & k \in \mathbb{Z} \cap I_{h} \\
c_{-k}^{-} \phi_{-k, j} & -k \in \mathbb{Z} \cap I_{h} \\
0 & k \notin \mathbb{Z} \cap I_{h}\end{cases} \\
\mu_{k}(h)= \begin{cases}\sqrt{\beta_{k}(h)} & k \in \mathbb{N} \\
-\sqrt{\beta_{-k}(h)} & -k \in \mathbb{N} .\end{cases}
\end{gathered}
$$

The components of $\vec{u}_{h}$, are then given by

$$
u_{h, j}=\sum_{k \in \mathbb{Z}} m_{k, j} \mathrm{e}^{i \mu_{k}(h) t}
$$

Thus,

$$
\begin{aligned}
u_{h, j}^{\prime} & =\sum_{k \in \mathbb{Z}} i \mu_{k, j}(h) m_{k, j} \mathrm{e}^{i \mu_{k}(h) t} \\
\left(A_{h}^{-1}{\overrightarrow{u_{h}}}^{\prime}\right)_{h, j} & =-\sum_{k \in \mathbb{Z}} i m_{k, j} \mathrm{e}^{i \mu_{k}(h) t} .
\end{aligned}
$$


Substituting identities (44) and (45) in (15) and having into account that the matrix $A_{h}=\frac{1}{h^{2}} A$ is orthogonal, we deduce that

$$
E^{h}(t)=h \sum_{j=0}^{N} \sum_{k \in \mathbb{N}}\left\{\left|c_{k}^{+}\right|^{2}\left|\frac{\phi_{k, j+1}-\phi_{k, j}}{h}\right|^{2}+\left|c_{k}^{-}\right|^{2}\left|\frac{\phi_{k, j+1}-\phi_{k, j}}{h}\right|^{2}\right\} .
$$

Moreover, from (46) and the identity (17) we have that

$$
E^{h}(t) \leq \frac{2}{4-\sqrt{\gamma}} \sum_{k \in \mathbb{N}}\left\{\left|c_{k}^{+}\right|^{2}+\left|c_{k}^{-}\right|^{2}\right\}\left|\frac{\phi_{k, N}}{h}\right|^{2}=\frac{2}{4-\sqrt{\gamma}} \sum_{k \in Z}\left|\frac{m_{k, N}}{h}\right|^{2} .
$$

From Lemma 2.2, for all $T>0$ exists $\delta_{0}>0$ and $k_{0} \in \mathbb{N}$, such that

$$
\mu_{k+1}(h)-\mu_{k}(h) \geq \frac{2 \pi}{T} \quad \forall k \in K_{0}(h)=\left\{k_{0}+1, k_{0}+2, \ldots, \frac{1-3 h}{h}-k_{0}\right\} \quad \text { with }|h| \leq \delta_{0} .
$$

Hence $Y=\left\{1,2, \ldots, k_{0}, \frac{1-2 h}{h}-k_{0}, \ldots, \frac{1-2 h}{h}\right\}$.

Let us observe that, independently of the size of $h$, the set of indices $Y$ is constituted by $2 k_{0}$ elements.

On the other hand, in (ii) of Lemma 2.1 it was proved that for all $\epsilon \in(0,1)$, there exists $\delta_{1}>0$, such that

$$
\mu_{k+1}(h)-\mu_{k}(h) \geq 3 \pi^{2}-\epsilon \quad \forall k \in \mathbb{Z} \text { and }|h|<\delta_{1} .
$$

Thus, applying Lemma 2.3 in (47) it is immediate to check that, for all $T>0$, with $\delta=\min \left\{\delta_{0}, \delta_{1}\right\}>0$, there exists a constant $C=C(T, \gamma)>0$, such that

$$
E^{h}(0) \leq C \int_{0}^{T}\left|\frac{u_{h, N}(t)}{h}\right|^{2} \mathrm{~d} t, \quad \forall \vec{u}_{h} \in \mathbf{C}_{h}(\gamma) \quad \text { with }|h|<\delta .
$$

This completes the proof of Theorem 2.2.

\subsection{Uniform observability by reinforced boundary measurements}

The goal of this section is to show that, in view of the gap properties obtained in the previous section, the observability inequality is uniform if the boundary measurement is reinforced in a suitable way.

Theorem 2.3. For all $T>0$, there exists $C=C(T)>0$ such that

$$
E^{h}(0) \leq C\left\{\int_{0}^{T}\left|\frac{u_{h, N}(t)}{h}\right|^{2} \mathrm{~d} t+h^{2} \int_{0}^{T}\left|\frac{u_{h, N}^{\prime}(t)}{h}\right|^{2} \mathrm{~d} t\right\}
$$

for all $\vec{u}_{h}$ solution of (7) and $0<h<1$.

Proof. Consider $\vec{u}_{h}$ with components as in (44). According to the identities (46) and (17),

$$
\begin{aligned}
E^{h}(0) & =h \sum_{j=1}^{N} \sum_{k \in \mathbb{N}}\left(\left|c_{k}^{+}\right|^{2}+\left|c_{k}^{-}\right|^{2}\right)\left|\frac{\phi_{k, j+1}-\phi_{k, j}}{h}\right|^{2}=\sum_{k \in \mathbb{N}} \frac{2}{\left(4-\left|\mu_{k}(h)\right| h^{2}\right)}\left(\left|c_{k}^{+}\right|^{2}+\left|c_{k}^{-}\right|^{2}\right)\left|\frac{\phi_{k, N}}{h}\right|^{2} \\
& =\sum_{k \in \mathbb{Z}^{*}} \frac{2}{\left(4-\left|\mu_{k}(h)\right| h^{2}\right)}\left|\frac{m_{k, N}}{h}\right|^{2} .
\end{aligned}
$$


On the other hand, from Theorems 2.2 and 2.1 we have that, for all $T>0$ there exists $\delta>0$, such that

$$
c_{1} \sum_{k \in \mathbb{Z}^{*}}\left|\frac{m_{k, N}}{h}\right|^{2} \geq \int_{0}^{T}\left|\frac{u_{h, N}(t)}{h}\right|^{2} \mathrm{~d} t \geq C_{1} \sum_{k \in \mathbb{Z}^{*}}\left|\frac{m_{k, N}}{h}\right|^{2},
$$

for all $|h|<\delta$.

Analogously,

$$
\begin{aligned}
c_{2} \sum_{k \in \mathbb{Z}^{*}}\left|\mu_{k}(h)\right|^{2}\left|\frac{m_{k, N}}{h}\right|^{2} & \geq \int_{0}^{T}\left|\frac{u_{h, N}^{\prime}(t)}{h}\right|^{2} \mathrm{~d} t=\int_{0}^{T}\left|\sum_{k \in \mathbb{Z}^{*}} i \mu_{k}(h) \mathrm{e}^{i \mu_{k}(h) t} \frac{m_{k, N}}{h}\right|^{2} \mathrm{~d} t \\
& \geq C_{2} \sum_{k \in \mathbb{Z}^{*}}\left|\mu_{k}(h)\right|^{2}\left|\frac{m_{k, N}}{h}\right|^{2} .
\end{aligned}
$$

Thus, for $\hat{C}=\min \left\{C_{2}, C_{1}\right\}$ :

$$
\begin{aligned}
\int_{0}^{T}\left|\frac{u_{h, N}(t)}{h}\right|^{2} \mathrm{~d} t+h^{2} \int_{0}^{T}\left|\frac{u_{h, N}^{\prime}(t)}{h}\right|^{2} \mathrm{~d} t & \geq \hat{C} \sum_{k \in \mathbb{Z}^{*}}\left(1+h^{2}\left|\mu_{k}(h)\right|^{2}\right)\left|\frac{m_{k, N}}{h}\right|^{2} \\
& \geq \hat{C} \sum_{k \in \mathbb{Z}^{*}}\left(1+h^{2}\left|\mu_{k}(h)\right|^{2}\right)\left\{\frac{4-\left|\mu_{k}(h)\right| h^{2}}{4-\left|\mu_{k}(h)\right| h^{2}}\right\}\left|\frac{m_{k, N}}{h}\right|^{2}
\end{aligned}
$$

On the other hand, for $h$ sufficiently small,

$$
\begin{aligned}
\left(1+h^{2}\left|\mu_{k}(h)\right|^{2}\right)\left(4-h^{2}\left|\mu_{k}(h)\right|\right) & =4\left\{1+\frac{16}{h^{2}} \sin ^{4}\left(\frac{|k| \pi h}{2}\right)\right\} \cos ^{2}\left(\frac{|k| \pi h}{2}\right) \\
& \geq 4\left\{\cos ^{2}\left(\frac{|k| \pi h}{2}\right)+k^{2} \pi^{2} \sin ^{2}\left(\frac{|k| \pi h}{2}\right)\right\} \geq 4, \quad \text { for }|k|=1,2, \ldots, 1 / h-1
\end{aligned}
$$

Therefore,

$$
\begin{aligned}
\int_{0}^{T}\left|\frac{u_{h, N}(t)}{h}\right|^{2} \mathrm{~d} t+h^{2} \int_{0}^{T}\left|\frac{u_{h, N}^{\prime}(t)}{h}\right|^{2} \mathrm{~d} t & \geq c \sum_{k \in \mathbb{Z}^{*}} \frac{1}{\left(4-\left|\mu_{k}(h)\right| h^{2}\right)}\left|\frac{m_{k, N}}{h}\right|^{2} \\
& \geq c h \sum_{k \in \mathbb{N}} \sum_{j=1}^{N}\left(\left|c_{k}^{+}\right|^{2}+\left|c_{k}^{-}\right|^{2}\right)\left|\frac{\phi_{k, j+1}-\phi_{k, j}}{h}\right|^{2} .
\end{aligned}
$$

This concludes the proof of Theorem 2.3.

\subsection{The reverse inequalities}

The following holds:

Proposition 2.1. For any $T>0$, there exists $c=c(T)>0$ such that

$$
E^{h}(0) \geq c \int_{0}^{T}\left|\frac{u_{h, N}(t)}{h}\right|^{2} \mathrm{~d} t, \quad \forall \vec{u}_{h}, \quad 0<h<1 .
$$


Proof. According to the identities (17) and (46), we have

$$
E^{h}(t)=\sum_{k \in \mathbb{Z}^{*}} \frac{2}{\left(4-\lambda_{k}(h) h^{2}\right)}\left|m_{k, h}(t)\right|^{2}\left|\frac{\phi_{k, N}}{h}\right|^{2} .
$$

As $4-\lambda_{k}(h) h^{2}=4 \cos ^{2}\left(\frac{k \pi h}{2}\right) \leq 4$,

$$
E^{h}(t) \geq \frac{1}{2} \sum_{k \in \mathbb{Z}^{*}}\left|m_{k, h}(t)\right|^{2}\left|\frac{\phi_{k, N}}{h}\right|^{2}
$$

Then, applying Lemma 2.3, taking (59) into account and properties (48) and (49) on the eigenvalues under consideration we deduce (58).

As an immediate consequence of Proposition 2.1 the following holds:

Proposition 2.2. For any $T>0$, there exists $c=c(T)>0$ such that

$$
E^{h}(0)+h^{2} E_{*}^{h}(0) \geq c\left\{\int_{0}^{T}\left|\frac{u_{h, N}(t)}{h}\right|^{2} \mathrm{~d} t+h^{2} \int_{0}^{T}\left|\frac{u_{h, N}^{\prime}(t)}{h}\right|^{2} \mathrm{~d} t\right\}, \forall \vec{u}_{h},
$$

where $E_{*}^{h}$ is the energy associated to $\vec{u}_{h}^{\prime}$ instead of $\vec{u}_{h}$, i.e.

$$
E_{*}^{h}(t)=\frac{h}{2} \sum_{j=0}^{N}\left\{\left|\frac{u_{h, j+1}^{\prime}-u_{h, j}^{\prime}}{h}\right|^{2}+\left|\frac{\left(A_{h}^{-1} \vec{u}_{h}^{\prime \prime}\right)_{h, j+1}-\left(A_{h}^{-1} \vec{u}_{h}^{\prime \prime}\right)_{h, j}}{h}\right|^{2}\right\}
$$

Remark 2.3. Due to the fact that $\left(\vec{u}_{h}^{\prime}\right)^{\prime}=\vec{u}_{h}^{\prime \prime}=-A_{h}^{2} \vec{u}_{h}$, the energy $E_{*}^{h}$ satisfies

$$
E_{*}^{h}(t)=\frac{h}{2} \sum_{j=0}^{N}\left\{\left|\frac{u_{h, j+1}^{\prime}-u_{h, j}^{\prime}}{h}\right|^{2}+\left|\frac{\left(A_{h} \vec{u}_{h}\right)_{h, j+1}-\left(A_{h} \vec{u}_{h}\right)_{h, j}}{h}\right|^{2}\right\} .
$$

\section{Control of the Semi-Discrete EQUATion}

In this section, we apply the observability results obtained above to analyze the controllability properties of the semi-discrete system.

\subsection{The semi-discrete control problem}

Let $\mathcal{P}=\left\{0=x_{0}<x_{1}<\cdots<x_{N+1}=1\right\}$. It is natural to introduce the following approximations of the boundary conditions in (6):

$$
\begin{aligned}
& y_{h, 0}=y_{h, N+1}=0 \\
& y_{h, 1}-2 y_{h, 0}+y_{h,-1}=0 \\
& y_{h, N+2}-2 y_{h, N+1}+y_{h, N}=h^{2} \nu_{h}(t) .
\end{aligned}
$$


Let us now consider the following controlled semi-discrete systems

$$
\left\{\begin{array}{l}
y_{h, j}^{\prime \prime}=-\frac{1}{h^{4}}\left\{y_{h, j+2}-4 y_{h, j+1}+6 y_{h, j}-4 y_{h, j-1}+y_{h, j-2}\right\} \\
0<t<T, \quad 1 \leq j \leq N \\
y_{h, 0}=y_{h, N+1}=0, \quad 0<t<T \\
y_{h,-1}=-y_{h, 1} \quad y_{h, N+2}=-y_{h, N}+h^{2} \nu_{h}, \quad 0<t<T \\
y_{h, j}(0)=y_{h, j}^{0} \quad y_{h, j}^{\prime}(0)=y_{h, j}^{1}, \quad j=1, \ldots, N .
\end{array}\right.
$$

This system may be viewed as a semi-discretization of (6) but also as the semi-discrete system (7) under the action of a control $\nu_{h}$.

We denote by $\vec{y}_{h}(t)=\left(y_{h, 1}(t), y_{h, 2}(t), \ldots, y_{h, N}(t)\right)$ the solution of $(64)$, with control $\nu_{h}$. Note that, $y_{h, j}^{0}$ and $y_{h, j}^{1}$ are, as usual, approximations of the initial conditions $\left(y^{0}, y^{1}\right)$ of the control problem $(6)$ at the points $x_{j}$.

\subsection{The discrete spaces $\mathcal{H}_{h}^{s}$ and $\mathcal{H}_{h, \gamma}^{s}$}

The eigenvectors $\vec{\phi}^{k}(h)$ of the spectral problem (8) satisfy

$$
h \sum_{j=1}^{N}\left|\phi_{k, j}(h)\right|^{2}=h \sum_{j=1}^{N} \sin ^{2}(j k \pi h)=1 / 2 .
$$

However, to simplify the notation, in what follows, we shall normalize them so that

$$
h\left|\vec{\phi}^{k}(h)\right|_{\mathbb{R}^{N}}^{2}=h \sum_{j=1}^{N}\left|\phi_{k, j}(h)\right|^{2}=1 .
$$

For every $s \in \mathbb{R}$, introduce the finite dimensional Hilbert spaces $\mathcal{H}_{h}^{s}=\operatorname{span}\left\{\vec{\phi}^{1}(h), \ldots, \vec{\phi}^{N}(h)\right\}$ endowed with the norm

$$
\left\|\vec{v}_{h}\right\|_{s, h}^{2}=\sum_{k=1}^{N} \lambda_{k}^{s}(h)\left|c_{k}\right|^{2}, \quad \text { whenever } \quad \vec{v}_{h}=\sum_{k=1}^{N} c_{k} \vec{\phi}^{k}(h) .
$$

In particular, $\mathcal{H}_{h}^{0}$ will be denoted by $L_{h}^{2}$.

Remark 3.1. The norm in $\mathcal{H}_{h}^{-1}$ is the dual to that in $\mathcal{H}_{h}^{1}$ in the sense that

$$
\left\|\vec{v}_{h}\right\|_{-1, h}=\sup _{\vec{w}_{h} \in \mathcal{H}_{h}^{1}} \frac{\left|\left(\vec{v}_{h}, \vec{w}_{h}\right)_{0, h}\right|}{\left\|\vec{w}_{h}\right\|_{1, h}}
$$

where $\left(\vec{v}_{h}, \vec{w}_{h}\right)_{0, h}=h \sum_{j=1}^{N} v_{h, j} w_{h, j}$ denotes the scalar product in $L_{h}^{2}$.

We also introduce the discrete energy space $\mathcal{F}_{h}=\mathcal{H}_{h}^{1} \times \mathcal{H}_{h}^{-1}$, with norm

$$
\left\|\left(\vec{u}_{h}, \vec{v}_{h}\right)\right\|_{\mathcal{F}_{h}}^{2}=\left\|\vec{u}_{h}\right\|_{1, h}^{2}+\left\|\vec{v}_{h}\right\|_{-1, h}^{2} .
$$

The dual of $\mathcal{F}_{h}$ is denoted by $\mathcal{F}_{h}^{*}$. 
Let us now introduce, for all $h>0$ and $0<\gamma<16$, the space generated by the eigenfunctions $\vec{\phi}^{k}(h)$ of the spectral problem (8) with indexes on the set $I_{h, \gamma}=\left\{1 \leq k \leq 1 / h-1: \lambda_{k}(h) \leq \sqrt{\gamma} h^{-2}\right\}$. In other words

$$
\mathcal{V}_{h, \gamma}=\operatorname{span}\left\{\vec{\phi}^{k}(h): k \in I_{h, \gamma}\right\}
$$

For every $s \in \mathbb{R}$, we denote by $\mathcal{H}_{h, \gamma}^{s}$ the subspace of $\mathcal{H}_{h}^{s}$ generated by the eigenvectors $\vec{\phi}^{k}(h)$ with eigenvalues lying in $I_{h, \gamma}$, endowed with the norm of $\mathcal{H}_{h}^{s}$, i.e.

$$
\left\|\vec{v}_{h}\right\|_{s, h}^{2}=\sum_{k \in I_{h, \gamma}} \lambda_{k}^{s}(h)\left|c_{k}\right|^{2}, \quad \text { whenever } \vec{v}_{h}=\sum_{k \in I_{h, \gamma}} c_{k} \vec{\phi}^{k}(h)
$$

\subsection{Partial controllability}

Let $\gamma \in(0,16)$ and $T>0$. The partial controllability problem of system (64) in the space $\mathcal{F}_{h}=\mathcal{H}_{h}^{1} \times \mathcal{H}_{h}^{-1}$ consists in finding a control $\nu_{h} \in L^{2}(0, T)$ such that the solution $\vec{y}_{h}$ of $(64)$ satisfies

$$
\Pi_{\gamma}\left(\vec{y}_{h}(T), \vec{y}^{\prime}(T)\right)=0
$$

where $\Pi_{\gamma}$ is the orthogonal projection from $\mathcal{F}_{h}$ into $\mathcal{F}_{h, \gamma}=\mathcal{H}_{h, \gamma}^{1} \times \mathcal{H}_{h, \gamma}^{-1}$ and

$$
\Pi_{\gamma}\left(\vec{y}_{h}(T), \overrightarrow{y h}^{\prime}(T)\right)=\left(\Pi_{\gamma} \vec{y}_{h}(T), \Pi_{\gamma} \overrightarrow{y h}^{\prime}(T)\right)=\left(\sum_{k \in I_{h, \gamma}} a_{k} \vec{\phi}^{k}(h), \sum_{k \in I_{h, \gamma}} b_{k} \vec{\phi}^{k}(h)\right),
$$

where $\left(a_{k}\right)$ and $\left(b_{k}\right)$ are the Fourier coefficients of $\left(\vec{y}_{h}(T), \vec{y}^{\prime} h(T)\right)$ in the basis of the eigenvectors $\left\{\vec{\phi}^{k}(h)\right\}_{k \in I_{h, \gamma}}$.

Multiplying (64) by $u_{h, j}$, adding in $j$, integrating in time, and using the symmetry of the matrix $B_{h}$ we get

$$
0=\sum_{j=1}^{N} \int_{0}^{T} y_{h, j}\left(u_{h, j}^{\prime \prime}+\left(B_{h} \vec{u}_{h}\right)_{h, j}\right) \mathrm{d} t+\left.\sum_{j=1}^{N}\left(y_{h, j}^{\prime} u_{h, j}-y_{h, j} u_{h, j}^{\prime}\right)\right|_{0} ^{T}+\int_{0}^{T} \frac{u_{h, N}(t) \nu_{h}(t)}{h^{2}} \mathrm{~d} t .
$$

Thus, for all solution $\vec{u}_{h}$ of (7), we have

$$
\sum_{j=1}^{N}\left(y_{h, j}^{\prime}(T) u_{h, j}(T)-y_{h, j}(T) u_{h, j}^{\prime}(T)\right)=\sum_{j=1}^{N}\left(y_{h, j}^{1} u_{h, j}^{0}-y_{h, j}^{0} u_{h, j}^{1}\right)-\int_{0}^{T} \frac{u_{h, N}(t) \nu_{h}(t)}{h^{2}} \mathrm{~d} t .
$$

Now, consider the functional $L_{T}: \mathbb{R}^{N} \times \mathbb{R}^{N} \rightarrow \mathbb{R}:$

$$
L_{T}\left({\overrightarrow{y_{h}}}^{0}, \overrightarrow{y h}^{1}\right)=\sum_{j=1}^{N}\left(y_{h, j}^{1} u_{h, j}^{0}-y_{h, j}^{0} u_{h, j}^{1}\right)-\int_{0}^{T} \frac{u_{h, N}(t) \nu_{h}(t)}{h^{2}} \mathrm{~d} t
$$

We obtain the following characterization of the partial controllability property of system (64).

Lemma 3.1. Let $T>0$ and $\gamma \in(0,16)$. Problem (64) is partially controllable in $\mathcal{F}_{h}=\mathcal{H}_{h}^{1} \times \mathcal{H}_{h}^{-1}$, iff for each 
$\left({\overrightarrow{y_{h}}}^{0},{\overrightarrow{y_{h}}}^{1}\right) \in \mathcal{F}_{h}$ there exists $\nu_{h} \in L^{2}(0, T)$, such that

$$
\sum_{j=1}^{N}\left(y_{h, j}^{1} u_{h, j}^{0}-y_{h, j}^{0} u_{h, j}^{1}\right)=\frac{1}{h^{2}} \int_{0}^{T} \nu_{h}(t) u_{h, N}(t) \mathrm{d} t
$$

for any initial data $\left({\overrightarrow{u_{h}}}^{0},{\overrightarrow{u_{h}}}^{1}\right)$ associated to solution $\vec{u}_{h} \in \mathbf{C}_{h}(\gamma)$.

Proof. It is immediate from (72).

The following uniform (with respect to $h \rightarrow 0$ ) partial controllability property holds in the space $\mathbf{C}_{h}(\gamma)$.

Theorem 3.1. For all $T>0$ and $\gamma \in(0,16)$ system (64) is partially controllable on $\mathcal{F}_{h}=\mathcal{H}_{h}^{1} \times \mathcal{H}_{h}^{-1}$ for all $0<h<1$. Moreover, there exists a constant $C=C(T, \gamma)>0$, independent of $0<h<1$, such that

$$
\left\|\nu_{h}\right\|_{L^{2}(0, T)} \leq C\left\|\Pi_{\gamma}\left({\overrightarrow{y_{h}}}^{1},-{\overrightarrow{y_{h}}}^{0}\right)\right\|_{\mathcal{F}_{h}^{*}}, \quad \forall 0<h<1
$$

for any $\left({\overrightarrow{y_{h}}}^{0},{\overrightarrow{y_{h}}}^{1}\right) \in \mathcal{F}_{h}$ where $\nu_{h}$ is the control of minimal $L^{2}(0, T)$-norm.

Proof. We define the functional $J_{h}: \mathbb{R}^{N} \times \mathbb{R}^{N} \rightarrow \mathbb{R}$, by

$$
J_{h}\left(\left({\overrightarrow{u_{h}}}^{0},{\overrightarrow{u_{h}}}^{1}\right)\right)=\frac{1}{2} \int_{0}^{T}\left|\frac{u_{h, N}(t)}{h}\right|^{2} \mathrm{~d} t-h \sum_{j=1}^{N}\left(y_{h, j}^{1} u_{h, j}^{0}-y_{h, j}^{0} u_{h, j}^{1}\right),
$$

where $\overrightarrow{u_{h}}$ is the solution of $(7)$ in the class $\mathbf{C}_{h}(\gamma)$ with initial data $\left({\overrightarrow{u_{h}}}^{0},{\overrightarrow{u_{h}}}^{1}\right)$.

We have

$$
\begin{aligned}
\left|h \sum_{j=1}^{N}\left(y_{h, j}^{1} u_{h, j}^{0}-y_{h, j}^{0} u_{h, j}^{1}\right)\right| & =\left|\left(\Pi_{\gamma}{\overrightarrow{y_{h}}}^{1},{\overrightarrow{u_{h}}}^{0}\right)_{\mathbb{R}^{N}}\right|+\left|\left(\Pi_{\gamma}{\overrightarrow{y_{h}}}^{0},-{\overrightarrow{u_{h}}}^{1}\right)_{\mathbb{R}^{N}}\right| \\
& \leq\left\|\Pi_{\gamma}{\overrightarrow{y_{h}}}^{1}\right\|_{-1, h}\left\|{\overrightarrow{u_{h}}}^{0}\right\|_{1, h}+\left\|\Pi_{\gamma}{\overrightarrow{y_{h}}}^{0}\right\|_{1, h}\left\|{\overrightarrow{u_{h}}}^{1}\right\|_{-1, h} \\
& \leq\left\|\Pi_{\gamma}\left({\overrightarrow{y_{h}}}^{1},-{\overrightarrow{y_{h}}}^{0}\right)\right\|_{\mathcal{F}_{h}^{*}}\left\|\left({\overrightarrow{u_{h}}}^{0},{\overrightarrow{u_{h}}}^{1}\right)\right\|_{\mathcal{F}_{h}} .
\end{aligned}
$$

According to (75) and the direct inequality in Proposition in 2.1 we deduce that $J_{h}$ is continuous for each $0<h<1$. Moreover $J_{h}$ is convex.

On the other hand, according to the observability inequality in Theorem $2.2, J_{h}$ is coercive in $\mathbf{C}_{h}(\gamma)$, uniformly on $0<h<1$.

Thus, for each $0<h<1$ there is a unique minimizer $\left({\overrightarrow{u_{h}}}^{0, *},{\overrightarrow{u_{h}}}^{1, *}\right)$ of the functional $J_{h}$ :

$$
J_{h}\left({\overrightarrow{u_{h}}}^{0, *},{\overrightarrow{u_{h}}}^{1, *}\right)=\min _{\left({\overrightarrow{u_{h}}}^{0},{\overrightarrow{u_{h}}}^{1}\right) \in \mathcal{F}_{h, \gamma}} J_{h}\left(\left({\overrightarrow{u_{h}}}^{0},{\overrightarrow{u_{h}}}^{1}\right)\right) .
$$

Calculating the Gateux derivative of $J_{h}$ in ${\left(\vec{u}_{h}\right.}^{0, *},{\overrightarrow{u_{h}}}^{1, *})$, we get

$$
\begin{aligned}
J_{h}^{\prime}\left(\left({\overrightarrow{u_{h}}}^{0, *},{\overrightarrow{u_{h}}}^{1, *}\right)\right) \cdot\left({\overrightarrow{u_{h}}}^{0},{\overrightarrow{u_{h}}}^{1}\right) & =\int_{0}^{T} \frac{u_{h, N}^{*}(t) u_{h, N}(t)}{h^{2}} \mathrm{~d} t-h \sum_{j=1}^{N}\left(y_{h, j}^{1} u_{h, j}^{0}-y_{h, j}^{0} u_{h, j}^{1}\right) \\
& =0, \quad \forall\left({\overrightarrow{u_{h}}}^{0},{\overrightarrow{u_{h}}}^{1}\right) \in \mathcal{F}_{h, \gamma} .
\end{aligned}
$$

According to $(73)$ and $(76), \nu_{h}=u_{h, N}^{*}(t) / h$ is the control we were looking for. 
Let us finally prove the uniform $L^{2}(0, T)$-bound on the controls. We have

$$
J_{h}\left(\left({\overrightarrow{u_{h}}}^{0, *}, \overrightarrow{u h}^{1, *}\right)\right) \leq J_{h}(0,0)=0
$$

Moreover, from (75) it follows that

$$
\frac{1}{2} \int_{0}^{T}\left|\frac{u_{h, N}^{*}(t)}{h}\right|^{2} \mathrm{~d} t \leq\left\|\Pi_{\gamma}\left({\overrightarrow{y_{h}}}^{1},-{\overrightarrow{y_{h}}}^{0}\right)\right\|_{\mathcal{F}_{h}^{*}}\left\|\left({\overrightarrow{u_{h}}}^{0, *},{\overrightarrow{u_{h}}}^{1, *}\right)\right\|_{\mathcal{F}_{h}}
$$

Combining (77) with the uniform observability inequality of Theorem 2.2 yields

$$
\frac{1}{2} \int_{0}^{T}\left|\frac{u_{h, N}^{*}(t)}{h}\right|^{2} \mathrm{~d} t \leq \hat{C}\left\|\Pi_{\gamma}\left({\overrightarrow{y_{h}}}^{1},-{\overrightarrow{y_{h}}}^{0}\right)\right\|_{\mathcal{F}_{h}^{*}}\left(\int_{0}^{T}\left|\frac{u_{h, N}^{*}(t)}{h}\right|^{2} \mathrm{~d} t\right)^{1 / 2},
$$

or, equivalently,

$$
\left\|\nu_{h}\right\|_{L^{2}(0, T)}^{2}=\int_{0}^{T}\left|\frac{u_{h, N}^{*}(t)}{h}\right|^{2} \mathrm{~d} t \leq C(T, \gamma)\left\|\Pi_{\gamma}\left(\overrightarrow{y h}^{1},-\overrightarrow{y h}^{0}\right)\right\|_{\mathcal{F}_{h}^{*}}^{2}
$$

where $C(T, \gamma)$ is independent of $0<h<1$ as we wanted to prove.

\subsection{Uniform exact controllability with reinforced controls}

In this section we analyze the problem of exact controllability. Thus, we look for controls such that the whole solution (and not only its projections $\Pi_{\gamma}$ ) vanishes at time $t=T$. This will be achieved, as in the previous section, minimizing a suitable quadratic functional with the aid of the uniform observability inequality of Theorem 3.1. The additional term we add on the functional reinforces the observed quantity on the boundary and leads to controls that are uniformly bounded in $H^{-1}(0, T)$ but not in $L^{2}(0, T)$. In fact, we will be able to obtain a sharper decomposition of the control but, as mentioned above, it will be naturally bounded in $H^{-1}(0, T)$.

Note that a control $\nu_{h} \in H^{-1}(0, T)$ such that the solution $\vec{y}_{h}$ of (64) satisfies

$$
\vec{y}_{h}(T)={\overrightarrow{y_{h}}}^{\prime}(T)=0, \quad \text { for all } h \in(0,1)
$$

may be characterized by the property that

$$
h \sum_{j=1}^{N}\left(y_{h, j}^{1} u_{h, j}^{0}-y_{h, j}^{0} u_{h, j}^{1}\right)=\left\langle\nu_{h}(t), \frac{u_{h, N}(t)}{h}\right\rangle_{H^{-1}(0, T) \times H^{1}(0, T)} .
$$

Observe that the duality between $H^{-1}(0, T)$ and $H^{1}(0, T)$ is not necessarily well defined. To avoid this difficulty we shall build $H^{-1}(0, T)$-controls with compact support in $(0, T)$.

We emphasize that, for any $h>0$, the controls $\nu_{h}$ we shall obtain are arbitrarily smooth since we are dealing with a linear finite dimensional control system but it is natural to work on the frame of $H^{-1}(0, T)$ to get uniform bounds, as $h \rightarrow 0$.

Theorem 3.2. For all $T>0$, system (64) is exactly controllable in time $T$, for all $h \in(0,1)$. Moreover, for all $\left(\overrightarrow{y h}^{0}, \overrightarrow{y h}^{1}\right) \in \mathcal{F}_{h}=\mathcal{H}_{h}^{1} \times \mathcal{H}_{h}^{-1}$ the control $\nu_{h}(t)$ may be found such that

$$
\nu_{h}(t)=\nu_{1, h}(t)+h \nu_{2, h}^{\prime}(t)
$$


where $\nu_{1, h}(t) \in L^{2}(0, T)$ and $\nu_{2, h}^{\prime}(t) \in H^{-1}(0, T)$ (with compact support on $(0, T)$ ) and so that

$$
\begin{gathered}
\left\|\nu_{1, h}\right\|_{L^{2}(0, T)} \leq C\left\|\left({\overrightarrow{y_{h}}}^{0},{\overrightarrow{y_{h}}}^{1}\right)\right\|_{\mathcal{F}_{h}}, \\
\left\|\nu_{2, h}^{\prime}\right\|_{H^{-1}(0, T)} \leq C\left\|\left({\overrightarrow{y_{h}}}^{0}, \overrightarrow{y h}^{1}\right)\right\|_{\mathcal{F}_{h}}
\end{gathered}
$$

with a constant $C>0$ which is independent of $0<h<1$ and the data $\left(\overrightarrow{y h}^{0}, \overrightarrow{y h}^{1}\right) \in \mathcal{F}_{h}$ to be controlled.

Proof. Given $T>0$ and $\epsilon>0$ small enough $(\epsilon<T / 2)$ we consider $\rho_{\epsilon}(t) \in C^{\infty}(\mathbb{R})$, so that $0 \leq \rho_{\epsilon} \leq 1, \rho_{\epsilon}=1$ in $(\epsilon, T-\epsilon)$ and the support of $\rho_{\epsilon}$ is compact in $(0, T)$.

For $\left({\overrightarrow{u_{h}}}^{0},{\overrightarrow{u_{h}}}^{1}\right) \in \mathcal{F}_{h}$, we define the quadratic functional $J_{h}: \mathbb{R}^{N} \times \mathbb{R}^{N} \rightarrow \mathbb{R}$ by

$$
J_{h}\left(\left({\overrightarrow{u_{h}}}^{0}, \overrightarrow{u h}^{1}\right)\right)=\frac{1}{2} \int_{0}^{T}\left|\frac{u_{h, N}(t)}{h}\right|^{2} \mathrm{~d} t+\frac{h^{2}}{2} \int_{0}^{T} \rho_{\epsilon}(t)\left|\frac{u_{h, N}^{\prime}(t)}{h}\right|^{2} \mathrm{~d} t-h \sum_{j=1}^{N}\left(y_{h, j}^{1} u_{h, j}^{0}-y_{h, j}^{0} u_{h, j}^{1}\right) .
$$

Observe that $J_{h}$ is continuous and convex. Moreover, according to Theorem 2.3, $J_{h}$ is also coercive in $\mathcal{F}_{h}$. Therefore, there exists a unique $\left({\overrightarrow{u_{h}}}^{0, *}, \overrightarrow{u h}^{1, *}\right) \in \mathcal{F}_{h}$ minimizing $J_{h}$.

On the other hand, we have

$$
\left|h \sum_{j=1}^{N}\left(y_{h, j}^{1} u_{h, j}^{0}-y_{h, j}^{0} u_{h, j}^{1}\right)\right| \leq\left\|\left({\overrightarrow{y_{h}}}^{1},-{\overrightarrow{y_{h}}}^{0}\right)\right\|_{\mathcal{F}_{h}^{*}}\left\|\left({\overrightarrow{u_{h}}}^{0},{\overrightarrow{u_{h}}}^{1}\right)\right\|_{\mathcal{F}_{h}} .
$$

Computing the Gateux derivative of $J_{h}$ at the minimizer we deduce that

$$
\begin{aligned}
& J_{h}^{\prime}\left(\left({\overrightarrow{u_{h}}}^{0, *},{\overrightarrow{u_{h}}}^{1, *}\right)\right) \cdot\left({\overrightarrow{u_{h}}}^{0},{\overrightarrow{u_{h}}}^{1}\right)=\int_{0}^{T} \frac{u_{h, N}^{*}(t) u_{h, N}(t)}{h^{2}} \mathrm{~d} t-h^{2}\left\langle\frac{\left(\rho_{\epsilon}\left(u_{h, N}^{*}\right)^{\prime}\right)^{\prime}}{h}, \frac{u_{h, N}}{h}\right\rangle_{H^{-1}(0, T) \times H^{1}(0, T)} \\
& +h \sum_{j=1}^{N}\left(u_{h, j}^{1} y_{h, j}^{0}-u_{h, j}^{0} y_{h, j}^{1}\right)=0, \quad \forall\left({\overrightarrow{u_{h}}}^{0},{\overrightarrow{u_{h}}}^{1}\right) \in \mathcal{F}_{h} .
\end{aligned}
$$

The control we are looking for is then $\nu_{h}(t)=\nu_{1, h}(t)+h \nu_{2, h}^{\prime}(t)$, with

$$
\nu_{1, h}(t)=\frac{u_{h, N}^{*}(t)}{h} \quad \text { and } \quad \nu_{2, h}(t)=-\rho_{\epsilon}(t)\left(u_{h, N}^{*}\right)^{\prime}(t) .
$$

On the other hand, $\left.J_{h}\left({\overrightarrow{u_{h}}}^{0, *},{\overrightarrow{u_{h}}}^{1, *}\right)\right) \leq J_{h}((0,0))=0$. Hence,

$$
\frac{1}{2} \int_{0}^{T}\left\{\left|\frac{u_{h, N}^{*}(t)}{h}\right|^{2}+h^{2} \rho_{\epsilon}(t)\left|\frac{\left(u_{h, N}^{*}\right)^{\prime}(t)}{h}\right|^{2}\right\} \mathrm{d} t \leq\left\langle{\overrightarrow{y_{h}}}^{1},{\overrightarrow{u_{h}}}^{0, *}\right\rangle_{\mathcal{H}_{h}^{-1} \times \mathcal{H}_{h}^{1}}+\left\langle{\overrightarrow{y_{h}}}^{0},-{\overrightarrow{u_{h}}}^{1, *}\right\rangle_{\mathcal{H}_{h}^{1} \times \mathcal{H}_{h}^{-1}} .
$$

Then, by Theorem 2.3, we get

$$
\begin{aligned}
& \frac{1}{2} \int_{0}^{T}\left|\frac{u_{h, N}^{*}(t)}{h}\right|^{2} \mathrm{~d} t+\frac{h^{2}}{2} \int_{0}^{T} \rho_{\epsilon}(t)\left|\frac{\left(u_{h, N}^{*}\right)^{\prime}(t)}{h}\right|^{2} \mathrm{~d} t \leq\left\|\left({\overrightarrow{y_{h}}}^{1},-{\overrightarrow{y_{h}}}^{0}\right)\right\|_{\mathcal{F}_{h}^{*}}\left\|\left({\overrightarrow{u_{h}}}^{0, *},{\overrightarrow{u_{h}}}^{1, *}\right)\right\|_{\mathcal{F}_{h}} \\
& \leq C\left\|\left({\overrightarrow{y_{h}}}^{0},{\overrightarrow{y_{h}}}^{1}\right)\right\|_{\mathcal{F}_{h}^{*}}\left(\frac{1}{2} \int_{0}^{T}\left|\frac{u_{h, N}^{*}(t)}{h}\right|^{2} \mathrm{~d} t+\frac{h^{2}}{2} \int_{0}^{T} \rho_{\epsilon}(t)\left|\frac{\left(u_{h, N}^{*}\right)^{\prime}(t)}{h}\right|^{2} \mathrm{~d} t\right)^{1 / 2} .
\end{aligned}
$$


Consequently

$$
\frac{1}{2} \int_{0}^{T}\left|\frac{u_{h, N}^{*}(t)}{h}\right|^{2} \mathrm{~d} t+\frac{h^{2}}{2} \int_{0}^{T} \rho_{\epsilon}(t)\left|\frac{\left(u_{h, N}^{*}\right)^{\prime}(t)}{h}\right|^{2} \mathrm{~d} t \leq C\left\|\left({\overrightarrow{y_{h}}}^{1},-{\overrightarrow{y_{h}}}^{0}\right)\right\|_{\mathcal{F}_{h}^{*}}^{2} .
$$

Therefore, $\frac{u_{h, N}^{*}(t)}{h}$ and $\rho_{\epsilon}(t)\left(u_{h, N}^{*}\right)^{\prime}(t)$ are uniformly bounded in $L^{2}(0, T)$, and

$$
h \nu_{2, h}^{\prime}=h^{2} \frac{\left(\rho_{\epsilon}(t)\left(u_{h, N}^{*}\right)^{\prime}(t)\right)^{\prime}}{h} \quad \text { is of the order of } h \text { in } H^{-1}(0, T) .
$$

This completes the proof of Theorem 3.2.

\section{Convergence Results}

In this section we study the convergence as $h \rightarrow 0$ of the semi-discrete systems without and with control.

We discuss both weak and strong convergence depending on the convergence properties of the initial data and the controls.

\subsection{Convergence for the uncontrolled semi-discrete system}

We consider families $\left\{\vec{u}_{h}(t)\right\}$ of solutions of (7) depending on the parameter $h$ and study their limit behavior as $h \rightarrow 0$. Recall that $\vec{u}_{h}$ admits the following development in Fourier series

$$
\vec{u}_{h}(t)=\sum_{k=1}^{N} m_{k, h}(t) \vec{\phi}^{k}(h)
$$

where

$$
m_{k, h}(t)=\left\{\begin{array}{lll}
c_{k}^{+} \mathrm{e}^{i \lambda_{k}(h) t} & \text { se } & k \in \mathbb{N} \\
c_{k}^{-} \mathrm{e}^{i \lambda_{k}(h) t} & \text { se } & k \notin \mathbb{N}
\end{array}\right.
$$

are the time-dependent Fourier coefficients.

Note that using the fact that $\sin \alpha / \alpha \geq c>0$, for all $0 \leq \alpha \leq \pi / 2$, for a suitable $c>0$ we have

$$
c k^{2} \pi^{2} \leq \lambda_{k}(h) \leq k^{2} \pi^{2} \quad \text { for all } \quad 0<h<1, \quad 1 \leq k \leq N .
$$

Let us now introduce, for every $s \in \mathbb{R}$, the following Hilbert spaces of sequences

$$
\mathcal{H}^{s}=\left\{\left\{c_{k}\right\}_{k \in \mathbb{N}} \in l^{2}: \sum_{k=1}^{\infty} k^{2 s}\left|c_{k}\right|^{2}<\infty\right\}
$$

endowed with their canonical norms. In particular $\mathcal{H}^{0}=l^{2}$.

Proposition 4.1. Let $\left\{\vec{u}_{h}(t)\right\}$ be a family of solutions of (7), depending on the parameter $h$, with uniformly bounded energies, i.e.

$$
E^{h}(0) \leq C \quad \text { for all } \quad h>0
$$


Then, by extracting a suitable subsequence $h \rightarrow 0$ we may guarantee that,

$$
\begin{aligned}
& m_{k, h}(.) \underset{h \rightarrow 0}{\longrightarrow} m_{k}(.) \quad \text { weakly in } \quad H^{1}(0, T), \quad \text { for all } \quad k \in \mathbb{N}, \\
& u(x, t)=\sum_{k \in \mathbb{N}} m_{k}(t) \sin (k \pi x) \quad \text { solves (1) for suitable initial data, } \\
& u \in C\left([0, T] ; H_{0}^{1}(0,1)\right) \cap C^{1}\left([0, T] ; H^{-1}(0,1)\right), \\
& E(0) \leq \liminf _{h \rightarrow 0} E^{h}(0), \\
& h \sum_{j=1}^{N}\left|u_{h, j}(t)\right|^{2} \underset{h \rightarrow 0}{\longrightarrow}|u(t)|_{L^{2}(0,1)}^{2} \text { in } L^{\infty}(0, T) .
\end{aligned}
$$

Proof. As an immediate consequence of (93) and from the conservation of energy we deduce that, for all $h \in(0,1)$,

$$
E^{h}(t)=\frac{h}{2} \sum_{j=0}^{N}\left\{\left|\frac{u_{h, j+1}-u_{h, j}}{h}\right|^{2}+\left|\frac{\left(A_{h}^{-1}{\overrightarrow{u_{h}}}^{\prime}\right)_{h, j+1}-\left(A_{h}^{-1}{\overrightarrow{u_{h}}}^{\prime}\right)_{h, j}}{h}\right|^{2}\right\} \leq C
$$

for all $0 \leq t \leq T$.

Due to the fact that the eigenvectors $\vec{\phi}^{k}(h)$ are orthogonal, we deduce that

$$
h \sum_{k=1}^{N}\left\{\left(\left|m_{k, h}(t)\right|^{2}+\lambda_{k}^{-2}(h)\left|m_{k, h}^{\prime}(t)\right|^{2}\right) \sum_{j=0}^{N}\left|\frac{\phi_{k, j+1}(h)-\phi_{k, j}(h)}{h}\right|^{2}\right\} \leq C .
$$

Since,

$$
h \sum_{j=1}^{N}\left|\frac{\phi_{k, j+1}-\phi_{k, j}}{h}\right|^{2}=h \lambda_{k}(h) \sum_{j=1}^{N}\left|\phi_{k, j}(h)\right|^{2}=\frac{\lambda_{k}(h)}{2}
$$

we conclude that, for all $h \in(0,1)$,

$$
E^{h}(0)=\frac{1}{2} \sum_{k=1}^{N}\left(\lambda_{k}(h)\left|m_{k, h}(t)\right|^{2}+\lambda_{k}^{-1}(h)\left|m_{k, h}^{\prime}(t)\right|^{2}\right) \leq C .
$$

Then, from the uniform boundedness estimate (91) we have

$$
\begin{aligned}
& \left\{\vec{m}_{h}(.)\right\}_{k \in \mathbb{N}} \quad \text { uniformly bounded in } \quad L^{\infty}\left(0, T ; \mathcal{H}^{1}\right) \\
& \left\{\vec{m}_{h}^{\prime}(.)\right\}_{k \in \mathbb{N}} \quad \text { uniformly bounded in } \quad L^{\infty}\left(0, T ; \mathcal{H}^{-1}\right)
\end{aligned}
$$

Thus, extracting subsequences, it follows that

$$
\begin{aligned}
& \vec{m}_{h}(.) \underset{h \rightarrow 0}{\rightarrow} \vec{m}(.) \quad \text { weakly } * \text { in } \quad L^{\infty}\left(0, T ; \mathcal{H}^{1}\right) \\
& \vec{m}_{h}^{\prime}(.) \underset{h \rightarrow 0}{\rightarrow} \vec{m}^{\prime}(.) \quad \text { weakly } * \text { in } \quad L^{\infty}\left(0, T ; \mathcal{H}^{-1}\right) .
\end{aligned}
$$


In particular,

$$
\begin{aligned}
& \vec{m}_{h}(.) \underset{h \rightarrow 0}{\rightarrow} \vec{m}(.) \quad \text { weakly in } \quad L^{2}\left(0, T ; \mathcal{H}^{1}\right) . \\
& \vec{m}_{h}^{\prime}(.) \underset{h \rightarrow 0}{\rightarrow} \vec{m}^{\prime}(.) \quad \text { weakly in } \quad L^{2}\left(0, T ; \mathcal{H}^{-1}\right) .
\end{aligned}
$$

On the other hand, due to the fact that

$$
m_{k, h}^{\prime \prime}+\lambda_{k}^{2}(h) m_{k, h}=0
$$

from (103) we have

$$
\sum_{k \in \mathbb{N}} \lambda_{k}^{-3}(h)\left|m_{k, h}^{\prime \prime}(t)\right|^{2}=\sum_{k \in \mathbb{N}} \lambda_{k}(h)\left|m_{k, h}(t)\right|^{2} \leq C, \quad \text { for all } h \in(0,1) .
$$

Consequently,

$$
\vec{m}_{h}^{\prime \prime}(.) \underset{h \rightarrow 0}{\rightarrow} \vec{m}^{\prime \prime}(.) \quad \text { weakly } * \text { in } \quad L^{\infty}\left(0, T ; \mathcal{H}^{-3}\right) .
$$

Using the classical Aubin-Lions compactness Lemma (see for instance Simon [14]), according to (105) and (111) we deduce that

$$
\vec{m}_{h}(.) \underset{h \rightarrow 0}{\longrightarrow} \vec{m}(.) \quad \text { strongly in } \quad C\left([0, T] ; \mathcal{H}^{s}\right), \quad \text { for all } \quad s<1
$$

and

$$
\vec{m}_{h}^{\prime}(.) \underset{h \rightarrow 0}{\longrightarrow} \vec{m}^{\prime}(.) \quad \text { strongly in } \quad C\left([0, T] ; \mathcal{H}^{-s}\right), \quad \text { for all } \quad s>1 .
$$

According to the bounds (105) and (106) and the convergence (112) and (113) we deduce that

$$
\begin{aligned}
& \vec{m}_{h}(0) \underset{h \rightarrow 0}{\rightarrow} \vec{m}^{0} \quad \text { weakly in } \mathcal{H}^{1}, \\
& \vec{m}_{h}^{\prime}(0) \underset{h \rightarrow 0}{\longrightarrow} \vec{m}^{1} \quad \text { weakly in } \quad \mathcal{H}^{-1},
\end{aligned}
$$

where

$$
\vec{m}(0)=\vec{m}^{0} \in \mathcal{H}^{1}, \quad \vec{m}^{\prime}(0)=\vec{m}^{1} \in \mathcal{H}^{-1} .
$$

Then, $u$ is defined as in (95), $u \in L^{\infty}\left(0, T ; H_{0}^{1}(0,1)\right) \cap W^{1, \infty}\left(0, T ; H^{-1}(0,1)\right)$ and, according to (105) and (106), it is the solution of (1) with initial data

$$
u^{0}(x)=\sum_{k \in \mathbb{N}} m_{k}^{0} \sin (k \pi x) \in H_{0}^{1}(0,1), u^{1}(x)=\sum_{k \in \mathbb{N}} m_{k}^{1} \sin (k \pi x) \in H^{-1}(0,1) .
$$

By uniqueness of solutions of (1) we deduce that (96) holds.

On the other hand, convergence (98) follows from (112) and (65). Indeed

$$
h \sum_{j=1}^{N}\left|u_{h, j}(t)\right|^{2}=\frac{1}{2} \sum_{k=1}^{N}\left|m_{k, h}(t)\right|^{2} \underset{h \rightarrow 0}{\longrightarrow} \frac{1}{2} \sum_{k=1}^{N}\left|m_{k}(t)\right|^{2}=|u(t)|_{L^{2}(0,1)}^{2}, \quad \text { in } C([0, T]) .
$$


We conclude this proof checking the property of lower semi-continuity of the energy (97). From (114) and (115) we get

$$
\begin{aligned}
& \sum_{k \in \mathbb{N}} k^{2} \pi^{2}\left|m_{k}(0)\right|^{2} \leq \liminf _{h \rightarrow 0} \sum_{k=1}^{N} \lambda_{k}(h)\left|m_{k, h}(0)\right|^{2} \\
& \sum_{k \in \mathbb{N}}\left(k^{2} \pi^{2}\right)^{-1}\left|m_{k}^{\prime}(0)\right|^{2} \leq \liminf _{h \rightarrow 0} \sum_{k=1}^{N} \lambda_{k}^{-1}(h)\left|m_{k, h}^{\prime}(0)\right|^{2} .
\end{aligned}
$$

Therefore, denoting $\lambda_{k}=k^{2} \pi^{2}$ we have

$$
\sum_{k \in \mathbb{N}}\left(\lambda_{k}\left|m_{k}(0)\right|^{2}+\lambda_{k}^{-1}\left|m_{k}^{\prime}(0)\right|^{2}\right) \leq \liminf _{h \rightarrow 0}\left\{\sum_{k \in \mathbb{N}} \lambda_{k}(h)\left|m_{k, h}(0)\right|^{2}+\sum_{k \in \mathbb{N}} \lambda_{k}^{-1}(h)\left|m_{k, h}^{\prime}(0)\right|^{2}\right\},
$$

and (97) holds.

\subsection{Convergence of the normal derivatives}

In the following propositions we study the convergence of the normal derivatives of the solution of the semi-discrete systems. These results will be important in the study of the convergence of the solutions of the controlled semi-discrete system.

Proposition 4.2. Let $\left\{\vec{u}_{h}(t)\right\}$ be a family of solutions of (7) depending on $h \rightarrow 0$ and satisfying (93). Let $u$ be any solution of (1) obtained as limit when $h \rightarrow 0$ of $\left\{\vec{u}_{h}(t)\right\}$ as in the statement of Proposition 4.1. Then

$$
\frac{u_{h, N}(t)}{h} \underset{h \rightarrow 0}{\rightarrow} \partial_{x} u(1, t) \text { weakly in } L^{2}(0, T),
$$

and therefore

$$
\int_{0}^{T}\left|\partial_{x} u(1, t)\right|^{2} \mathrm{~d} t \leq \liminf _{h \rightarrow 0} \int_{0}^{T}\left|\frac{u_{h, N}(t)}{h}\right|^{2} \mathrm{~d} t .
$$

Proof. From inequality (58) and (93) we have

$$
\int_{0}^{T}\left|\frac{u_{h, N}(t)}{h}\right|^{2} \mathrm{~d} t=\left|\sum_{k \in \mathbb{N}} m_{k, h}(t) \frac{\phi_{k, N}(h)}{h}\right|_{L^{2}(0, T)}^{2} \leq C \quad \forall h \in(0,1) .
$$

Hence, there exists a subsequence of $\{h\}_{0<h<1}$, such that

$$
\nu_{h}=\frac{u_{h, N}(t)}{h} \underset{h \rightarrow 0}{\rightarrow} \nu \quad \text { weakly in } \quad L^{2}(0, T) .
$$

On the other hand, from (112) it follows that

$$
m_{k, h}(.) \underset{h \rightarrow 0}{\longrightarrow} m_{k}(.) \quad \text { strongly in } \quad C([0, T]), \quad \text { for all } k \in \mathbb{N} .
$$

Moreover, for all $k \in \mathbb{N}$,

$$
\frac{\phi_{k, N}(h)}{h}=\frac{\sin (N k \pi h)}{h}=\frac{\sin k(1-h) \pi}{h}=-\cos (k \pi) \frac{\sin (k \pi h)}{h}
$$


converges to

$$
-\left.\partial_{x} \phi^{k}\right|_{x=1}=-\left.\partial_{x}(\sin (k \pi x))\right|_{x=1}=-\cos (k \pi) k \pi,
$$

when $h \longrightarrow 0$.

Therefore, combining $(124,125)$ and $(126)$ we obtain that

$$
-\left.m_{k, h}(.) \frac{\phi_{k, N}(h)}{h} \underset{h \rightarrow 0}{\longrightarrow} m_{k}(.) \partial_{x} \phi^{k}\right|_{x=1} \quad \text { strongly in } \quad C([0, T]), \quad \forall k \in \mathbb{N} .
$$

Let $u(x, t)=\sum_{k \in \mathbb{N}} m_{k}(t) \sin (k \pi x)$ be the limit solution of (1). It is sufficient to check that the weak limit $\nu$ in (123) coincides with $\partial_{x} u(1, t)$.

For any test function $\varphi \in \mathcal{D}(0, T)$ we set

$$
\begin{aligned}
& \vec{v}_{h}=\sum_{k=1}^{N}\left\langle m_{k, h}, \varphi\right\rangle_{\mathcal{D}^{\prime}(0, T) \times \mathcal{D}(0, T)} \vec{\phi}^{k}(h) \\
& v(x)=\sum_{k \in \mathbb{N}}\left\langle m_{k}, \varphi\right\rangle_{\mathcal{D}^{\prime}(0, T) \times \mathcal{D}(0, T)} \sin (k \pi x) .
\end{aligned}
$$

It is then sufficient to check that

$$
\frac{v_{h, N}}{h} \underset{h \rightarrow 0}{\longrightarrow} \partial_{x} v(1)
$$

At this respect note that,

$$
\left(B_{h} \vec{u}_{h}\right)_{h, j}=-u_{h, j}^{\prime \prime}=-\sum_{k=1}^{N} m_{k, h}^{\prime \prime}(t) \phi_{k, j}(h)
$$

and

$$
\partial_{x}^{4} u(x)=-u^{\prime \prime}(x)=-\sum_{k \in \mathbb{N}} m_{k}^{\prime \prime}(t) \partial_{x}^{4} \phi^{k}(x)
$$

Consequently,

$$
\left(B_{h} \vec{v}_{h}\right)_{h, j}=\sum_{k=1}^{N}\left\langle m_{k, h}, \varphi\right\rangle_{\mathcal{D}^{\prime}(0, T) \times \mathcal{D}(0, T)}\left(B_{h} \vec{\phi}^{k}(h)\right)_{h, j}=-\sum_{k=1}^{N}\left\langle m_{k, h}^{\prime \prime}, \varphi\right\rangle_{\mathcal{D}^{\prime}(0, T) \times \mathcal{D}(0, T)} \phi_{k, j}(h),
$$

and

$$
\partial_{x}^{4} v(x)=\sum_{k \in \mathbb{N}}\left\langle m_{k}, \varphi\right\rangle_{\mathcal{D}^{\prime}(0, T) \times \mathcal{D}(0, T)} \partial_{x}^{4} \phi^{k}(x)=-\sum_{k \in \mathbb{N}}\left\langle m_{k}^{\prime \prime}, \varphi\right\rangle_{\mathcal{D}^{\prime}(0, T) \times \mathcal{D}(0, T)} \phi^{k}(x) .
$$


Consider the elliptic problem

$$
\left\{\begin{array}{l}
-\partial_{x}^{4} v=g \quad \text { in }(0,1) \\
v(0)=v(1)=0 \\
\partial_{x}^{2} v(0)=\partial_{x}^{2} v(1)=0
\end{array}\right.
$$

where $g(x)=\sum_{k \in \mathbb{N}} g^{k} \sin (k \pi x)$ with $g^{k}=\left\langle m_{k}^{\prime \prime}, \varphi\right\rangle_{\mathcal{D}^{\prime}(0, T) \times \mathcal{D}(0, T)}$. From $(134)$ we have $g \in H^{-1}(0,1)$.

On the other hand, let us consider the discretized problems

$$
\left\{\begin{array}{l}
-\left(B_{h} \vec{v}_{h}\right)_{h, j}=g_{h, j}, \quad j=1,2, \ldots, N \\
v_{h, 0}=v_{h, N+1}=0 \\
v_{h,-1}=-v_{h, 1} ; \quad v_{h, N+2}=-v_{h, N}
\end{array}\right.
$$

where $g_{h, j}=\sum_{k=1}^{N} g_{h}^{k} \sin (j k \pi h)$ with $g_{h}^{k}=\left\langle m_{k, h}^{\prime \prime}, \varphi\right\rangle_{\mathcal{D}^{\prime}(0, T) \times \mathcal{D}(0, T)}$.

Observe that, from (107) and (108) we have

$$
\left\{m_{k, h}^{\prime \prime}(.)\right\}_{k \in \mathbb{N}} \underset{h \rightarrow 0}{\longrightarrow}\left\{m_{k}^{\prime \prime}(.)\right\}_{k \in \mathbb{N}} \quad \text { weakly in } \quad H^{-1}\left(0, T ; \mathcal{H}^{-1}\right) .
$$

Consequently,

$$
\left\{g_{h}^{k}\right\}_{k=1}^{N} \underset{h \rightarrow 0}{\rightarrow}\left\{g^{k}\right\}_{k \in \mathbb{N}} \quad \text { weakly in } \quad \mathcal{H}^{-1}
$$

On the other hand, the solutions $v_{h, j}$ of (136) and $v$ of (135), are given by

$$
v_{h, j}=-\sum_{k=1}^{N} g_{h}^{k} \frac{\sin (k \pi h j)}{\lambda_{k}^{2}(h)}, \quad j=1,2, \ldots, N
$$

and

$$
v=-\sum_{k \in \mathbb{N}} g^{k} \frac{\sin (k \pi x)}{\lambda_{k}^{2}}
$$

Convergence (130) is equivalent to proving that

$$
\sum_{k=1}^{N} \frac{g_{h}^{k}}{\lambda_{k}^{2}(h)} \frac{\sin (k \pi N h)}{h}=\sum_{k=1}^{N} \frac{g_{h}^{k}}{\lambda_{k}^{2}(h)} \frac{\sin (k \pi h)}{h} \cos (k \pi) \underset{h \rightarrow 0}{\longrightarrow} \sum_{k \in \mathbb{N}} \frac{g^{k}}{k^{3} \pi^{3}} \cos (k \pi),
$$

or, taking into account that the convergence of each term of the series for $k$ fixed holds, for any $\epsilon>0$ there exists $M_{\epsilon}>0$, such that

$$
\left|\sum_{k \geq M_{\epsilon}}\left(\frac{g_{h}^{k}}{\lambda_{k}^{2}(h)} \frac{\sin (k \pi h)}{h}-\frac{g^{k}}{k^{3} \pi^{3}}\right) \cos (k \pi)\right| \leq \epsilon
$$


Taking into account that $\left\{g^{k}\right\}_{k \in \mathbb{N}} \in \mathcal{H}^{-1}$, it is easy to see that, for sufficiently large $M_{\epsilon}$ :

$$
\begin{aligned}
\left|\sum_{k \geq M_{\epsilon}} \frac{g^{k}}{k^{3} \pi^{3}} \cos (k \pi)\right| & \leq \frac{1}{\pi^{3}}\left(\sum_{k \geq M_{\epsilon}} k^{-2}\left|g^{k}\right|^{2}\right)^{1 / 2}\left(\sum_{k \geq M_{\epsilon}} k^{-4} \cos ^{2}(k \pi)\right)^{1 / 2} \\
& \leq \frac{C}{\pi^{3}}\left(\sum_{k \geq M_{\epsilon}} \frac{1}{k^{4}}\right)^{1 / 2} \leq \frac{\epsilon}{2}
\end{aligned}
$$

Similarly, since $c k^{2} \leq \lambda_{k}(h)$ we have

$$
\begin{aligned}
\left|\sum_{k \geq M_{\epsilon}} \frac{g_{h}^{k}}{\lambda_{k}^{2}(h)} \frac{\sin (k \pi h)}{h} \cos (k \pi)\right| & \leq \sum_{k \geq M_{\epsilon}} \frac{\left|g_{h}^{k}\right|}{\lambda_{k}^{2}(h)} k \pi \leq\left(\sum_{k \geq M_{\epsilon}} \frac{\left|g_{h}^{k}\right|^{2}}{\lambda_{k}(h)}\right)^{1 / 2}\left(\sum_{k \geq M_{\epsilon}} \frac{k^{2} \pi^{2}}{\lambda_{k}^{3}(h)}\right)^{1 / 2} \\
& \leq C\left(\sum_{k \geq M_{\epsilon}} k^{-2}\left|g_{h}^{k}\right|^{2}\right)^{1 / 2}\left(\sum_{k \geq M_{\epsilon}} \frac{1}{k^{4}}\right)^{1 / 2} \leq \frac{\epsilon}{2}
\end{aligned}
$$

for a sufficiently large $M_{\epsilon}$, independent of $h$.

This completes proof of (139) and that of Proposition 4.2 as well.

The following result provides the strong convergence of the normal derivative under suitable assumptions on the initial data.

Proposition 4.3. Let $T>0$ and $h \in(0,1)$. Let $\left({\overrightarrow{u_{h}}}^{0}, \vec{u}_{h}{ }^{1}\right) \in \mathcal{H}_{h}^{1} \times \mathcal{H}_{h}^{-1}$ and $\left(u^{0}, u^{1}\right) \in H_{0}^{1}(0,1) \times H^{-1}(0,1)$ be the initial conditions of problems (7) and (1), given by

$$
\begin{aligned}
& {\overrightarrow{u_{h}}}^{0}=\sum_{k=1}^{N} m_{k, h}^{0} \vec{\phi}^{k}(h),{\overrightarrow{u_{h}}}^{1}=\sum_{k=1}^{N} m_{k, h}^{1} \vec{\phi}^{k}(h), \\
& u^{0}(x)=\sum_{k \in \mathbb{N}} m_{k}^{0} \phi^{k}(x), u^{1}(x)=\sum_{k \in \mathbb{N}} m_{k}^{1} \phi^{k}(x) .
\end{aligned}
$$

Assuming that $m_{k, h}^{0}=m_{k, h}^{1}=0$ for all $k>N$ we suppose that

$$
\begin{array}{lll}
\left\{m_{k, h}^{0}\right\}_{k \in \mathbb{N}} \underset{h \rightarrow 0}{\longrightarrow}\left\{m_{k}^{0}\right\}_{k \in \mathbb{N}} & \text { strongly in } & \mathcal{H}^{1}, \\
\left\{m_{k, h}^{1}\right\}_{k \in \mathbb{N}} \underset{h \rightarrow 0}{\longrightarrow}\left\{m_{k}^{1}\right\}_{k \in \mathbb{N}} & \text { strongly in } \mathcal{H}^{-1} .
\end{array}
$$

Then,

$$
\frac{u_{h, N}(t)}{h} \underset{h \rightarrow 0}{\longrightarrow} \partial_{x} u(1, t) \quad \text { strongly in } \quad L^{2}(0, T) .
$$

Proof. As a consequence of Proposition 4.2 we have

$$
\frac{u_{h, N}(t)}{h} \underset{h \rightarrow 0}{\rightarrow} \partial_{x} u(1, t) \quad \text { weakly in } \quad L^{2}(0, T),
$$

where

$$
\frac{u_{h, N}(t)}{h}=\sum_{k=1}^{N} m_{k, h}(t) \frac{\phi_{k, N}}{h}=\sum_{k=1}^{N} m_{k, h}(t) \frac{\sin (k \pi h)}{h} \cos (k \pi)
$$


and

$$
\partial_{x} u(1, t)=\left.\sum_{k \in \mathbb{N}} m_{k}(t) \partial_{x} \phi^{k}\right|_{x=1}=\sum_{k \in \mathbb{N}} k \pi m_{k}(t) \cos (k \pi) .
$$

To conclude the proof of (144) it is sufficient to show that the following holds:

$$
\int_{0}^{T}\left|\sum_{k=1}^{N} m_{k, h}(t) \frac{\sin (k \pi h)}{h} \cos (k \pi)-\sum_{k \in \mathbb{N}} k \pi m_{k}(t) \cos (k \pi)\right|^{2} \mathrm{~d} t \underset{h \rightarrow 0}{\longrightarrow} 0 .
$$

Thus, it is sufficient to check that, for every $\epsilon>0$, there exists $M_{\epsilon}>0$ such that

$$
\int_{0}^{T}\left|\sum_{k \geq M_{\epsilon}}\left(m_{k, h}(t) \frac{\sin (k \pi h)}{h}-m_{k}(t) k \pi\right) \cos (k \pi)\right|^{2} \mathrm{~d} t \leq \epsilon .
$$

In view of the convergence $(142,143)$ and the conservation of the energies $E^{h}$ we deduce that:

$$
\vec{m}_{h}(t)=\left\{m_{k, h}(t)\right\}_{k \in \mathbb{N}} \underset{h \rightarrow 0}{\longrightarrow} \vec{m}(t)=\left\{m_{k}(t)\right\}_{k \in \mathbb{N}} \quad \text { strongly in } \quad \mathcal{H}^{1},
$$

for all $0 \leq t \leq T$.

As a consequence of the direct inequality proved in Proposition 2.1 we deduce that

$$
\int_{0}^{T}\left|\sum_{k \geq M} m_{k, h}(t) \frac{\sin (k \pi h)}{h} \cos (k \pi)\right|^{2} \mathrm{~d} t \leq C_{0} \sum_{k \geq M}\left|m_{k, h}\right|^{2} k^{2} \pi^{2}, \quad \forall M \geq 1 .
$$

Due to the conservation of energy and taking hypotheses (142) and (143) into account we have that

$$
\sum_{k \geq M_{\epsilon}}\left|m_{k, h}\right|^{2} k^{2} \pi^{2} \leq \frac{\epsilon}{2}, \quad \text { for } M_{\epsilon} \text { large enough. }
$$

On the other hand, by the same argument,

$$
\int_{0}^{T}\left|\sum_{k \geq M_{\epsilon}}(k \pi) m_{k}(t) \cos (k \pi)\right|^{2} \mathrm{~d} t \leq \hat{C}_{0} \sum_{k \geq M_{\epsilon}}\left|m_{k}\right|^{2} k^{2} \pi^{2} \leq \frac{\epsilon}{2} .
$$

Combining (150) with $(148,149)$ convergence (146) follows immediately.

\subsection{Weak convergence of the semi-discrete problems with boundary control}

In this section we study the convergence of the solutions of the controlled systems (64). In view of the two uniform controllability results of Theorems 3.1 and 3.2 we distinguish two cases:

Theorem 4.1. Let $T>0$, and $0<\gamma<16$. For any $h \in(0,1)$, consider $\quad\left({\overrightarrow{y_{h}}}^{0},{\overrightarrow{y_{h}}}^{1}\right) \in \mathcal{H}_{h, \gamma}^{1} \times \mathcal{H}_{h, \gamma}^{-1} \quad$ and $\left(y^{0}, y^{1}\right) \in H_{0}^{1}(0,1) \times H^{-1}(0,1)$, the initial conditions in (64) and (6) respectively, given by

$$
{\overrightarrow{y_{h}}}^{0}=\sum_{k=1}^{N} a_{k, h}^{0} \vec{\phi}^{k}(h),{\overrightarrow{y_{h}}}^{1}=\sum_{k=1}^{N} b_{k, h}^{1} \vec{\phi}^{k}(h), y^{0}=\sum_{k \in \mathbb{N}} a_{k}^{0} \phi^{k}, y^{1}=\sum_{k \in \mathbb{N}} b_{k}^{1} \phi^{k} .
$$


Assuming that,

$$
\begin{aligned}
& \left\{a_{k, h}^{0}\right\}_{k \in \mathbb{N}} \underset{h \rightarrow 0}{\longrightarrow}\left\{a_{k}^{0}\right\}_{k \in \mathbb{N}} \text { weakly in } \mathcal{H}^{1}, \\
& \left\{b_{k, h}^{1}\right\}_{k \in \mathbb{N}} \underset{h \rightarrow 0}{\rightarrow}\left\{b_{k}^{1}\right\}_{k \in \mathbb{N}} \text { weakly in } \mathcal{H}^{-1},
\end{aligned}
$$

then, $\left(\vec{y}_{h}(t), \overrightarrow{y h}^{\prime}(t)\right) \in \mathcal{H}_{h, \gamma}^{1} \times \mathcal{H}_{h, \gamma}^{-1}$ and its partial controls $\nu_{h} \in L^{2}(0, T)$ are given as in Theorem 3.1. Moreover, the Fourier coefficients $a_{k, h}($.$) and b_{k, h}($.$) of \vec{y}_{h}$ and ${\overrightarrow{y^{\prime}}}_{h}$ respectively, are such that

$$
\begin{aligned}
& \left\{a_{k, h}(.)\right\}_{k \in \mathbb{N}} \underset{h \rightarrow 0}{\rightarrow}\left\{a_{k}(.)\right\}_{k \in \mathbb{N}} \quad \text { weakly *in } L^{\infty}\left(0, T ; \mathcal{H}^{1}\right), \\
& \left\{b_{k, h}(.)\right\}_{k \in \mathbb{N}} \underset{h \rightarrow 0}{\rightarrow}\left\{b_{k}(.)\right\}_{k \in \mathbb{N}} \quad \text { weakly } * \text { in } L^{\infty}\left(0, T ; \mathcal{H}^{-1}\right), \\
& \nu_{h}(.) \underset{h \rightarrow 0}{\rightarrow} \nu(.) \quad \text { weakly in } L^{2}(0, T),
\end{aligned}
$$

where, $\left(y(x, t), y^{\prime}(x, t)\right)=\left(\sum_{k \in \mathbb{N}} a_{k}(t) \phi^{k}(x), \sum_{k \in \mathbb{N}} b_{k}(t) \phi^{k}(x)\right) \quad$ solves (6), with control $\nu(t)$, and $y(T)=y^{\prime}(T)$ $=0$. The limit control $\nu$ is given by

$$
\nu(t)=-\partial_{x} u^{*}(1, t)
$$

where $u^{*}$ is solution of (1), with data $\left(u^{0, *}, u^{1, *}\right) \in H_{0}^{1}(0,1) \times H^{-1}(0,1)$ minimizing the functional

$$
J\left(\left(u^{0}, u^{1}\right)\right)=\frac{1}{2} \int_{0}^{T}\left|\partial_{x} u(1, t)\right|^{2} \mathrm{~d} t-\left\langle\left(y^{1}, y^{0}\right) ;\left(u^{0},-u^{1}\right)\right\rangle_{F^{\prime} \times F}
$$

in $H_{0}^{1}(0,1) \times H^{-1}(0,1)$.

We consider now as initial datum for the semi-discrete equation (64) $\left(\vec{y}_{h}^{0}, \vec{y}_{h}^{1}\right) \in \mathcal{H}_{h}^{1} \times \mathcal{H}_{h}^{-1}$. From Theorem 3.2 we obtain that the corresponding solutions and controls of (64) can be expressed by

$$
\vec{y}_{h}(t)=\vec{z}_{h}(t)+h \vec{w}_{h}(t) \quad \text { and } \quad \nu_{h}(t)=\nu_{1, h}(t)+h \nu_{2, h}^{\prime}(t)
$$

where $\vec{z}_{h}=\sum_{k=1}^{N} c_{k, h} \vec{\phi}^{k}(h)$ is the solution of (64) with control $\nu_{1, h} \in L^{2}(0, T)$ and initial data $\left({\overrightarrow{y_{h}}}^{0},{\overrightarrow{y_{h}}}^{1}\right)$, and $\vec{w}_{h}=\sum_{k=1}^{N} e_{k, h} \vec{\phi}^{k}(h)$ solves (64) with control $\nu_{2, h}^{\prime} \in H^{-1}(0, T)$ and zero initial data.

Theorem 4.2. Let $T>0$. For any $h \in(0,1)$, consider the initial data $\left(\vec{y}_{h}^{0}, \vec{y}_{h}^{1}\right) \in \mathcal{H}_{h}^{1} \times \mathcal{H}_{h}^{-1} \quad$ and $\left(y^{0}, y^{1}\right) \in H_{0}^{1}(0,1) \times H^{-1}(0,1)$ of problems (64) and (6) as in (151).

Assume that,

$$
\begin{aligned}
& \left\{a_{k, h}^{0}\right\}_{k \in \mathbb{N}} \underset{h \rightarrow 0}{\longrightarrow}\left\{a_{k}^{0}\right\}_{k \in \mathbb{N}} \text { weakly in } \mathcal{H}^{1} \\
& \left\{b_{k, h}^{1}\right\}_{k \in \mathbb{N}} \underset{h \rightarrow 0}{\rightarrow}\left\{b_{k}^{1}\right\}_{k \in \mathbb{N}} \text { weakly in } \mathcal{H}^{-1} .
\end{aligned}
$$


Then, the controls $\nu_{h}$, and the controlled solutions $\vec{y}_{h}$ in (158) satisfy

$$
\begin{aligned}
& \left\{c_{k, h}\right\}_{k \in \mathbb{N}} \underset{h \rightarrow 0}{\rightarrow}\left\{c_{k}\right\}_{k \in \mathbb{N}} \quad \text { weakly }{ }^{*} \text { in } L^{\infty}\left(0, T ; \mathcal{H}^{1}\right) \cap W^{1, \infty}\left(0, T ; \mathcal{H}^{-1}\right) \\
& h\left\{e_{k, h}\right\}_{k \in \mathbb{N}} \text { is of order } h \text { in } \quad L^{\infty}\left(0, T ; \mathcal{H}^{-1}\right) \cap W^{1, \infty}\left(0, T ; \mathcal{H}^{-3}\right), \\
& \nu_{1, h} \underset{h \rightarrow 0}{\rightarrow} \nu \text { weakly in } L^{2}(0, T), \\
& h \nu_{2, h}^{\prime} \text { is of order } h \text { in } H^{-1}(0, T),
\end{aligned}
$$

where $y(x, t)=\sum_{k \in \mathbb{N}} c_{k}(t) \phi^{k}(x)$ is solution of problem (6) with control $\nu(t)=-\partial_{x} u^{*}(1, t) \in L^{2}(0, T)$ such that $y(T)=y^{\prime}(T)=0$ and $u^{*}$ solves (1) with the initial data $\left(u^{0, *}, u^{1, *}\right)$ that minimizes the functional $J$ defined in (157) in $H_{0}^{1}(0,1) \times H^{-1}(0,1)$.

Proof of Theorem 4.1. We divide the proof in several steps.

First Step (Convergence of the Controls). In view of Theorem 3.1 and estimate (79), there exists a subsequence $\left\{\nu_{h}\right\}_{h}$, such that

$$
\nu_{h}(t)=\frac{u_{h, N}^{*}(t)}{h} \rightarrow \nu(t) \quad \text { in } \quad L^{2}(0, T), \quad \text { as } \quad h \rightarrow 0 .
$$

Recall that ${\overrightarrow{u_{h}}}^{*}(t)$ solves $(7)$ with data $\left({\overrightarrow{u_{h}}}^{0, *},{\overrightarrow{u_{h}}}^{1, *}\right) \in \mathcal{H}_{h, \gamma}^{1} \times \mathcal{H}_{h, \gamma}^{-1}$ minimizing the functional $J_{h}$ defined in $(74)$.

Moreover, as a consequence of the observability inequality (23) we have

$$
\left\|\left({\overrightarrow{u_{h}}}^{0, *},{\overrightarrow{u_{h}}}^{1, *}\right)\right\|_{\mathcal{F}_{h}} \leq C \text { for all } h>0 .
$$

In these conditions, Proposition 4.2 guarantees that $\nu(t)=\partial_{x} u^{*}(1, t)$, where $u^{*}$ solves (1).

Second Step. The solution $\vec{y}_{h}$ of (64) satisfies

$$
\begin{aligned}
\left\langle{\overrightarrow{y_{h}}}^{\prime}(s), \vec{u}_{h}(s)\right\rangle_{\mathcal{H}_{h}^{-1} \times \mathcal{H}_{h}^{1}}-\left\langle\vec{y}_{h}(s),{\overrightarrow{u_{h}}}^{\prime}(s)\right\rangle_{\mathcal{H}_{h}^{1} \times \mathcal{H}_{h}^{-1}}= & \left\langle{\overrightarrow{y_{h}}}^{1},{\overrightarrow{u_{h}}}^{0}\right\rangle_{\mathcal{H}_{h}^{-1} \times \mathcal{H}_{h}^{1}}-\left\langle{\overrightarrow{y_{h}}}^{0},{\overrightarrow{u_{h}}}^{1}\right\rangle_{\mathcal{H}_{h}^{-1} \times \mathcal{H}_{h}^{1}} \\
& +\int_{0}^{s} \nu_{h}(t) \frac{u_{h, N}(t)}{h} \mathrm{~d} t,
\end{aligned}
$$

for any $\vec{u}_{h} \in \mathbf{C}_{h}(\gamma)$ and all $0<s<T$.

According to Proposition 2.1 and estimate (166), we obtain that

$$
\left.\left|\left({\overrightarrow{y_{h}}}^{\prime}(s),-\overrightarrow{y_{h}}(s)\right)\left\|_{\mathcal{F}_{h}^{*}} \leq C\right\|\left({\overrightarrow{y_{h}}}^{1},-{\overrightarrow{y_{h}}}^{0}\right) \|_{\mathcal{F}_{h}^{*}}+\right| \nu_{h}\right|_{L^{2}(0, T)}, \quad \forall s \in(0, T) .
$$

Combining $(152,153,165)$ and $(167)$, we deduce the existence of a subsequence of indexes $\{h\}$, such that

$$
\begin{array}{lll}
\left\{a_{k, h}(t)\right\}_{k \in N} \underset{h \rightarrow 0}{\rightarrow}\left\{a_{k}(t)\right\}_{k \in \mathbb{N}} & \text { weakly } * \text { in } & L^{\infty}\left(0, T ; \mathcal{H}^{1}\right), \\
\left\{b_{k, h}(t)\right\}_{k \in N} \underset{h \rightarrow 0}{\rightarrow}\left\{b_{k}(t)\right\}_{k \in \mathbb{N}} & \text { weakly } * \text { in } & L^{\infty}\left(0, T ; \mathcal{H}^{-1}\right),
\end{array}
$$

where $\quad b_{k, h}(t)=a_{k, h}^{\prime}(t) \quad$ and $\quad b_{k}(t)=a_{k}^{\prime}(t)$.

Let us now prove that

$$
\left\{a_{k, h}^{\prime \prime}(t)\right\}_{k \in N} \underset{h \rightarrow 0}{\rightarrow}\left\{a_{k}^{\prime \prime}(t)\right\}_{k \in \mathbb{N}} \quad \text { weakly in } \quad L^{2}\left(0, T ; \mathcal{H}^{-3}\right)
$$


For that it is sufficient to show that ${\overrightarrow{y_{h}}}^{\prime \prime}$ is bounded in $L^{2}\left(0, T ; \mathcal{H}^{-3}\right)$ or, equivalently, for any $\vec{\theta}_{h} \in L^{2}\left(0, T ; \mathcal{H}_{h}^{3}\right)$

$$
\left|\left\langle\vec{y}^{\prime \prime}(t), \vec{\theta}_{h}(t)\right\rangle_{L^{2}\left(0, T ; \mathcal{H}_{h}^{-3}\right) \times L^{2}\left(0, T ; \mathcal{H}_{h}^{3}\right)}\right| \leq C\left\|\vec{\theta}_{h}\right\|_{L^{2}\left(0, T ; \mathcal{H}_{h}^{3}\right)}, \quad \forall h>0 .
$$

In fact, by multiplying (64) by $\theta_{h, j}(t)=\sum_{k=1}^{N} g_{k, h}(t) \phi_{k, j}$ (the $j$-th component of $\vec{\theta}_{h}$ ), adding in $j$ and integrating in $(0, T)$, we get that

$$
\begin{aligned}
\left|h \sum_{j=1}^{N} \int_{0}^{T} y_{h, j}^{\prime \prime} \theta_{h, j} \mathrm{~d} t\right|= & \left|h \sum_{j=1}^{N} \int_{0}^{T}\left(B_{h} \vec{y}_{h}\right)_{h, j} \theta_{h, j} \mathrm{~d} t\right| \\
= & \left|-\int_{0}^{T} \frac{\theta_{h, N}(t)}{h} \nu_{h} \mathrm{~d} t+h \sum_{j=1}^{N} \int_{0}^{T} y_{h, j}\left(B_{h} \vec{\theta}_{h}\right)_{h, j} \mathrm{~d} t\right| \\
\leq & \left|\int_{0}^{T} \frac{\theta_{h, N}}{h} \nu_{h} \mathrm{~d} t\right|+\left|\int_{0}^{T} \sum_{k=1}^{N} a_{k, h}(t) \lambda_{k}^{2}(h) g_{k, h}(t) \mathrm{d} t\right| \\
\leq & \left(\int_{0}^{T}\left\|\left\{a_{k, h}(t)\right\}\right\|_{\mathcal{H}^{1}}^{2} \mathrm{~d} t\right)^{1 / 2}\left(\int_{0}^{T}\left\|\left\{g_{k, h}(t)\right\}\right\|_{\mathcal{H}^{3}}^{2} \mathrm{~d} t\right)^{1 / 2} \\
& +\left|\frac{\theta_{h, N}(t)}{h}\right|_{L^{2}(0, T)}\left|\nu_{h}\right|_{L^{2}(0, T)} .
\end{aligned}
$$

On the other hand, due to $\theta_{h}(t) \in L^{2}\left(0, T ; \mathcal{H}_{h}^{3}\right)$, the sequence of Fourier coefficients $\left\{g_{k, h}(t)\right\}_{k \in \mathbb{N}} \in L^{2}\left(0, T ; \mathcal{H}^{3}\right)$ and

$$
\begin{aligned}
\left|\frac{\theta_{h, N}(t)}{h}\right|_{L^{2}(0, T)}^{2} & =\int_{0}^{T}\left|\sum_{k \in \mathbb{N}} g_{k, h} \frac{\sin (k \pi N h)}{h}\right|^{2} \mathrm{~d} t \\
& \leq\left(\sum_{k \in \mathbb{N}} \lambda_{k}^{-3}(h) \frac{\sin ^{2}(k \pi h)}{h^{2}}\right) \int_{0}^{T}\left(\sum_{k \in \mathbb{N}} \lambda_{k}^{3}(h)\left|g_{k, h}\right|^{2}\right) \mathrm{d} t \\
& \leq C \sum_{k \in \mathbb{N}} \frac{1}{k^{4}}\left\|\vec{\theta}_{h}\right\|_{L^{2}\left(0, T ; \mathcal{H}_{h}^{3}\right)}^{2}
\end{aligned}
$$

Therefore, as a consequence of inequality (172) the proof of (171) and, therefore, that of (170) finishes.

Then, from $(168,169)$ and $(170)$ we get

$$
\left\{a_{k}(t)\right\}_{k \in \mathbb{N}} \in C\left([0, T] ; \mathcal{H}^{-1}\right) \quad \text { and } \quad\left\{a_{k}^{\prime}(t)\right\}_{k \in \mathbb{N}} \in C\left([0, T] ; \mathcal{H}^{-3}\right),
$$

which implies the existence of $\left\{a_{k}(0)\right\}_{k \in \mathbb{N}}$ and $\left\{a_{k}^{\prime}(0)\right\}_{k \in \mathbb{N}}$, which are uniformly bounded in $\mathcal{H}^{1}$ and $\mathcal{H}^{-1}$ respectively.

Moreover, from $(168,169)$ and $(169,170)$ and Aubin-Lions compactness lemma, we deduce that

$$
\left\{a_{k, h}(t)\right\} \underset{h \rightarrow 0}{\longrightarrow}\left\{a_{k}(t)\right\}, \quad \text { strongly in } \quad C\left([0, T] ; \mathcal{H}^{\delta}\right) \cap C^{1}\left([0, T] ; \mathcal{H}^{-\delta}\right),
$$


for any $\delta>0$. Thus,

$$
\begin{aligned}
& \left\{a_{k, h}(0)\right\} \underset{h \rightarrow 0}{\rightarrow}\left\{a_{k}(0)\right\}, \quad \text { weakly in } \mathcal{H}^{1}, \\
& \left\{a_{k, h}^{\prime}(0)\right\} \underset{h \rightarrow 0}{\longrightarrow}\left\{a_{k}^{\prime}(0)\right\}, \quad \text { weakly in } \mathcal{H}^{-1} .
\end{aligned}
$$

Observe that, by the hypotheses of theorem, $a_{k}(0)=a_{k}^{0}$ and $a_{k}^{\prime}(0)=a_{k}^{1}$.

We also observe that, from (174), for each $k$ fixed, the following convergence holds

$$
\begin{aligned}
& a_{k, h}(t) \mathrm{e}^{i \lambda_{k, h}(t)} \underset{h \rightarrow 0}{\longrightarrow} a_{k}(t) \mathrm{e}^{i \lambda_{k}(t)}, \quad \text { for all } t \in[0, T] \\
& a_{k, h}^{\prime}(t) \mathrm{e}^{i \lambda_{k, h}(t)} \underset{h \rightarrow 0}{\longrightarrow} a_{k}^{\prime}(t) \mathrm{e}^{i \lambda_{k}(t)}, \quad \text { for all } t \in[0, T] .
\end{aligned}
$$

Third Step. Consider $\vec{u}_{h}=\mathrm{e}^{i \lambda_{p}(h) t} \vec{\phi}^{p}(h) \in \mathbf{C}_{h}(\gamma)$. Then, obviously,

$$
\frac{u_{h, N}(t)}{h} \longrightarrow \partial_{x} u(1, t) \quad \text { uniformly in } \quad[0, T]
$$

where $u(x, t)=\mathrm{e}^{i \lambda_{p} t} \boldsymbol{\phi}^{p}(x)$. From (165) it then follows that

$$
\int_{0}^{T} \nu_{h}(t) \frac{u_{h, N}(t)}{h} \mathrm{~d} t \longrightarrow \int_{0}^{T} \nu(t) \partial_{x} u(1, t) \mathrm{d} t, \quad \text { as } \quad h \rightarrow 0 .
$$

On the other hand, taking into account that $\vec{u}_{h} \rightarrow u$ in $C\left([0, T] ; \mathcal{H}^{1}\right)$ and that $(174)$ is valid for $\delta=1$, we have that the first term in the left hand side of (166) satisfies, for all $s \in(0, T)$,

$$
h \sum_{j=1}^{N} y_{h, j}^{\prime}(s) u_{h, j}(s) \underset{h \rightarrow 0}{\longrightarrow} \int_{0}^{1} y^{\prime}(x, s) u(x, s) \mathrm{d} x
$$

where $y(x, t)=\sum_{k \in \mathbb{N}} a_{k}(t) \phi^{k}(x)$

Similarly, in each term of (166) using the estimates (174) and (180) together with the fact of ${\overrightarrow{u^{\prime}}}_{h} \rightarrow u^{\prime}$ in $C\left([0, T] ; \mathcal{H}^{-1}\right)$ we show that when $h$ tends to zero,

$$
\begin{aligned}
\int_{0}^{1}\left\{y^{\prime}(x, s) u(x, s)-y(x, s) u^{\prime}(x, s)\right\} \mathrm{d} x= & \int_{0}^{1}\left\{y^{1}(x) u^{0}(x)-y^{0}(x) u^{1}(x)\right\} \mathrm{d} x \\
& -\int_{0}^{s} \nu(t) \partial_{x} u(1, t) \mathrm{d} t, \quad \forall s \in(0, T) .
\end{aligned}
$$

Thus, $y$ is the solution by transposition of (6) with control $\nu$.

Since $\Pi_{\gamma} \vec{y}_{h}(T)=\Pi_{\gamma}{\overrightarrow{y_{h}}}^{\prime}(T)=0$, by the convergences $(177,178)$ and the fact that, as $h \rightarrow 0$, all the Fourier components are eventually involved in the projections $\Pi_{\gamma}$, we deduce that $y(T)=y^{\prime}(T)=0$.

Step 4. To conclude the proof of theorem it is sufficient to show that $\nu=\partial_{x} u^{*}(1, t)$, where $u^{*}$ solves (1) with the initial data minimizing the functional (157). 
We know that both $\nu$ and $\partial_{x} u^{*}(1, t)$ are exact controls for the system $(6)$. Then

$$
\int_{0}^{T}\left(\nu(t)-\partial_{x} u^{*}(1, t)\right) \partial_{x} u(1, t) \mathrm{d} t=0
$$

for every solution $u$ of the adjoint system (1).

From Proposition 4.2 we have also that $\nu(t)=\partial_{x} z(1, t) \in L^{2}(0, T)$, where $z$ is solution a (1). Consequently

$$
\int_{0}^{T}\left(\partial_{x} z(1, t)-\partial_{x} u^{*}(1, t)\right) \partial_{x} u(1, t) \mathrm{d} t=0 .
$$

Taking $u=z-u^{*}$ as test function in this identity it follows that $\nu(t)=\partial_{x} u^{*}(1, t)$.

Proof of Theorem 4.2. We divide the proof in 3 steps.

Step 1. From Theorem 3.2 it follows that the control of system (64) has the form $\nu_{h}(t)=\nu_{1, h}(t)+h \nu_{2, h}^{\prime}(t)$. Moreover, from $(88,159)$ and $(160)$ we have that

$$
\begin{aligned}
& \left\{\nu_{1, h}(t)\right\}_{h} \quad \text { is uniformly bounded in } \quad L^{2}(0, T) \\
& \left\{\nu_{2, h}(t)\right\}_{h} \quad \text { is uniformly bounded in } L^{2}(0, T) \\
& \text { with compact support in }(0, T) \\
& \left\{\nu_{2, h}^{\prime}(t)\right\}_{h} \quad \text { is uniformly bounded in } H^{-1}(0, T) \\
& h \nu_{2, h}^{\prime}(t) \quad \text { is of order } h \text { in } \quad H^{-1}(0, T) \text {. }
\end{aligned}
$$

Hence, there exists a subsequence of $\{h\}$ such that

$$
\begin{array}{lll}
\nu_{1, h}(t) \underset{h \rightarrow 0}{\longrightarrow} \nu_{1}(t) & \text { weakly in } & L^{2}(0, T) \\
h \nu_{2, h}^{\prime}(t) \underset{h \rightarrow 0}{\longrightarrow} 0 & \text { strongly in } & H^{-1}(0, T) .
\end{array}
$$

Remark that, from Proposition 4.2, $\nu(t)=\partial_{x} u^{*}(1, t) \in L^{2}(0, T)$, where $u^{*}$ solves (1).

Step 2. Let $t \in[0, T]$ and $j=1, \ldots, N$. We introduce the following semi-discrete problems:

$$
\left\{\begin{array}{l}
z_{h, j}^{\prime \prime}=-\frac{1}{h^{4}}\left\{z_{h, j+2}-4 z_{h, j+1}+6 z_{h, j}-4 z_{h, j-1}+z_{h, j-2}\right\} \\
z_{h, 0}=z_{h, N+1}=0 \\
z_{h,-1}=-z_{h, 1} \quad z_{h, N+2}=-z_{h, N}+h^{2} \nu_{1, h} \\
z_{h, j}(0)=y_{h, j}^{0}, \quad z_{h, j}^{\prime}(0)=y_{h, j}^{1}
\end{array}\right.
$$

and

$$
\left\{\begin{array}{l}
w_{h, j}^{\prime \prime}=-\frac{1}{h^{4}}\left\{w_{h, j+2}-4 w_{h, j+1}+6 w_{h, j}-4 w_{h, j-1}+w_{h, j-2}\right\} \\
w_{h, 0}=w_{h, N+1}=0 \\
w_{h,-1}=-w_{h, 1} \quad w_{h, N+2}=-w_{h, N}+h^{2} \nu_{2, h}^{\prime} \\
w_{h, j}(0)=0, \quad w_{h, j}^{\prime}(0)=0
\end{array}\right.
$$


It is easy to see that the solution $\vec{y}_{h}(t)$ of (64) can be decomposed as $\vec{y}_{h}(t)=\vec{z}_{h}(t)+h \vec{w}_{h}(t)$. In particular, $\vec{z}_{h}$ satisfies the identity:

$$
h \sum_{j=1}^{N}\left(z_{h, j}^{\prime}(s) u_{h, j}(s)-z_{h, j}(s) u_{h, j}^{\prime}(s)\right)=h \sum_{j=1}^{N}\left(y_{h, j}^{1} u_{h, j}^{0}-y_{h, j}^{0} u_{h, j}^{1}\right)-\int_{0}^{T} \nu_{1, h}(t) \frac{u_{h, N}(t)}{h} \mathrm{~d} t
$$

for any $\vec{u}_{h}$ solution of (7). Now, taking Proposition 2.1 into account, we obtain that

$$
\begin{aligned}
& \left|\left\langle\left({\overrightarrow{z_{h}}}^{\prime}(s),-\vec{z}_{h}(s)\right),\left(\vec{u}_{h}(s),{\overrightarrow{u_{h}}}^{\prime}(s)\right)\right\rangle_{\mathcal{F}_{h}^{*} \times \mathcal{F}_{h}}\right| \\
& \leq\left\|\left(\vec{y}_{h}^{1},-\vec{y}_{h}^{0}\right)\right\| \mathcal{F}_{h}^{*}\left\|\left(\vec{u}_{h}^{0}, \vec{u}_{h}^{1}\right)\right\|_{\mathcal{F}_{h}}+\left|\nu_{1, h}(.)\right|_{L^{2}(0, T)}\left|\frac{u_{h, N}(t)}{h}\right|_{L^{2}(0, T)} \\
& \leq\left\|\left(\vec{u}_{h}^{0}, \vec{u}_{h}^{1}\right)\right\|_{\mathcal{F}_{h}}\left(\left\|\left(\vec{y}_{h}^{1},-\vec{y}_{h}^{0}\right)\right\|_{\mathcal{F}_{h}^{*}}+\left|\nu_{1, h}(.)\right|_{L^{2}(0, T)}\right)
\end{aligned}
$$

Since the sequence $\left\{\nu_{1, h}\right\}_{h}$ is uniformly bounded in $L^{2}(0, T)$, from the hypotheses (159) and (160) we have

$$
\left\|\left({\overrightarrow{z_{h}}}^{\prime}(s),-\vec{z}_{h}(s)\right)\right\|_{\mathcal{F}_{h}^{*}} \leq C\left\|\left(\vec{u}_{h}^{1},-\vec{u}_{h}^{0}\right)\right\|_{\mathcal{F}_{h}^{*}} \quad \text { for all } \quad s \in(0, T)
$$

Hence, $\left\{c_{k, h}(s)\right\}_{h}$, the sequence of Fourier coefficients of $\vec{z}_{h}$, is uniformly bounded in $L^{\infty}(0, T$, $\left.\mathcal{H}^{1}\right) \cap W^{1, \infty}\left(0, T, \mathcal{H}^{-1}\right)$.

On the other hand, we denote by $\vec{\varphi}_{h}$ the solution of the semi-discrete system:

$$
\left\{\begin{array}{l}
\varphi_{h, j}^{\prime \prime}=-\frac{1}{h^{4}}\left\{\varphi_{h, j+2}-4 \varphi_{h, j+1}+6 \varphi_{h, j}-4 \varphi_{h, j-1}+\varphi_{h, j-2}\right\} \\
\varphi_{h, 0}=\varphi_{h, N+1}=0, \quad 0<t<T \\
\varphi_{h,-1}=-\varphi_{h, 1} \quad \varphi_{h, N+2}=-\varphi_{h, N}+h^{2} \nu_{2, h} \\
\varphi_{h, j}(0)=0 \quad \varphi_{h, j}^{\prime}(0)=0
\end{array}\right.
$$

where $0<t<T$ and $1 \leq j \leq N$.

The sequence $\vec{\varphi}_{h}$ satisfies the same uniform boundedness properties of $\vec{z}_{h}$ above.

Taking into account that $\vec{w}_{h}={\overrightarrow{\varphi_{h}}}^{\prime}$, its Fourier coefficients $\left\{e_{k, h}(s)\right\}_{h}$ are uniformly bounded in $L^{\infty}(0, T$, $\left.\mathcal{H}^{-1}\right) \cap W^{1, \infty}\left(0, T, \mathcal{H}^{-3}\right)$

Step 3. From the previous estimates it follows that

$$
\begin{aligned}
& c_{k, h}(s) \underset{h \rightarrow 0}{\longrightarrow} c_{k}(s) \quad \text { weakly } * \text { in } \quad L^{\infty}\left(0, T, \mathcal{H}^{1}\right) \cap W^{1, \infty}\left(0, T, \mathcal{H}^{-1}\right) \\
& h e_{k, h}(s) \underset{h \rightarrow 0}{\longrightarrow} 0 \quad \text { strongly in } \quad L^{\infty}\left(0, T, \mathcal{H}^{-1}\right) \cap W^{1, \infty}\left(0, T, \mathcal{H}^{-3}\right) .
\end{aligned}
$$

But, since

$$
\begin{aligned}
& a_{k, h}(T)=c_{k, h}(T)+h e_{k, h}(T)=0 \\
& b_{k, h}(T)=c_{k, h}^{\prime}(T)+h e_{k, h}^{\prime}(T)=0,
\end{aligned}
$$

and $a_{k, h}(T)=b_{k, h}(T)$ for all $k$, we deduce that $\left(c_{k, h}(T), c_{k, h}^{\prime}(T)\right) \underset{h \rightarrow 0}{\longrightarrow}(0,0)$, as $h \rightarrow 0$, for all $k$.

As a consequence of (193) it follows that $c_{k}(T)=c_{k}^{\prime}(T)=0$. Therefore, the limit $y(x, t)=\sum_{k \in \mathbb{N}} c_{k}(t) \phi^{k}(x)$ of $\vec{z}_{h}$ solves (6) with control $\nu(t)=\partial_{x} u^{*}(1, t)$ and satisfies $y(T)=y^{\prime}(T)=0$.

Finally, using the same arguments of Theorem 4.1 we conclude that $u^{*}$ solves (1) with initial datum $\left(u^{0, *}, u^{1, *}\right)$ minimizing the functional defined in (74). 


\subsection{Strong convergence of the semi-discrete problem with boundary control}

Assuming the strong convergence of the initial data further convergence properties of controls and solutions can be proved:

Theorem 4.3. Let $T>0$ and $0<\gamma<16$. For any $h \in(0,1)$, consider $\left(\vec{y}_{h}^{0}, \vec{y}_{h}^{1}\right) \in \mathcal{H}_{h, \gamma}^{1} \times \mathcal{H}_{h, \gamma}^{-1} \quad$ and $\left(y^{0}, y^{1}\right) \in H_{0}^{1}(0,1) \times H^{-1}(0,1)$, the initial conditions in (64) and (6) as in (151).

Assuming that,

$$
\begin{aligned}
& \left\{a_{k, h}^{0}\right\}_{k \in \mathbb{N}} \underset{h \rightarrow 0}{\longrightarrow}\left\{a_{k}^{0}\right\}_{k \in \mathbb{N}} \quad \text { strongly in } \mathcal{H}^{1}, \\
& \left\{b_{k, h}^{1}\right\}_{k \in \mathbb{N}} \underset{h \rightarrow 0}{\longrightarrow}\left\{b_{k}^{1}\right\}_{k \in \mathbb{N}} \text { strongly in } \mathcal{H}^{-1},
\end{aligned}
$$

then, the partial controls $\nu_{h}(t) \in L^{2}(0, T)$, and the controlled solutions $\vec{y}_{h}$ satisfy

$$
\begin{aligned}
& \left\{a_{k, h}(t)\right\}_{k \in \mathbb{N}} \underset{h \rightarrow 0}{\longrightarrow}\left\{a_{k}(t)\right\}_{k \in \mathbb{N}} \quad \text { strongly in } \mathcal{H}^{1}, \quad \forall t \in(0, T) ; \\
& \left\{b_{k, h}(t)\right\}_{k \in \mathbb{N}} \underset{h \rightarrow 0}{\longrightarrow}\left\{b_{k}(t)\right\}_{k \in \mathbb{N}} \quad \text { strongly in } \mathcal{H}^{-1}, \quad \forall t \in(0, T) ; \\
& \nu_{h}(.) \underset{h \rightarrow 0}{\longrightarrow} \nu(.) \quad \text { strongly in } L^{2}(0, T) ;
\end{aligned}
$$

where, $\left(y(x, t), y^{\prime}(x, t)\right)=\left(\sum_{k \in \mathbb{N}} a_{k}(t) \phi^{k}(x), \sum_{k \in \mathbb{N}} b_{k}(t) \phi^{k}(x)\right) \quad$ solves $(6)$, with control $\nu(t) \in L^{2}(0, T)$, and $y(T)=y^{\prime}(T)=0$. Moreover, the control $\nu$ is given by

$$
\nu(t)=-\partial_{x} u^{*}(1, t)
$$

where $u^{*}$ is solution of (1), with initial data $\left(u^{0, *}, u^{1, *}\right)$ minimizing the functional defined in (157).

Proof. Combining Theorem 4.1 and Theorem 2.2 we obtain the weak convergence of the initial data $\left({\overrightarrow{u_{h}}}^{0, *},{\overrightarrow{u_{h}}}^{1, *}\right)$ $\in \mathcal{H}_{h, \gamma}^{1} \times \mathcal{H}_{h, \gamma}^{-1}$ of $(7)$, minimizing the functional $J_{h}$ defined in (74). Moreover, by the hypotheses of Theorem 4.3, the linear term of the functional $J_{h}$ converges to the linear term of the functional $J$ defined in (157). Therefore, proving (200) is equivalent to proving that

$$
J_{h}\left({\overrightarrow{u_{h}}}^{0, *},{\overrightarrow{u_{h}}}^{1, *}\right) \underset{h \rightarrow 0}{\longrightarrow} J\left(u^{0, *}, u^{1, *}\right)
$$

where $\left(u^{0, *}, u^{1, *}\right) \in H_{0}^{1}(0,1) \times H^{-1}(0,1)$ minimizes the functional $J$. Indeed, if this is true, taking the convergence of the linear term into account and the structure of the functionals $J_{h}$ and $J$ we deduce that

$$
\int_{0}^{T}\left|\nu_{h}(t)\right|^{2} \mathrm{~d} t \longrightarrow \int_{0}^{T}|\nu(t)|^{2} \mathrm{~d} t
$$

which, combined with the weak convergence property provides the desired strong convergence result.

In view of the weak convergence of the initial data and the controls, and by weak lower semicontinuity it is easy to see that

$$
J\left(u^{0, *}, u^{1, *}\right) \leq \liminf J_{h}\left({\overrightarrow{u_{h}}}^{0, *},{\overrightarrow{u_{h}}}^{1, *}\right) .
$$

Thus, in order to complete the proof of $(201)$ it is sufficient to check that for all $\left(u^{0}, u^{1}\right) \in \mathcal{D}$ where $\mathcal{D}$ is the subspace of initial data with a number finite of Fourier coefficients, which is dense in the space $H_{0}^{1}(0,1) \times$ $H^{-1}(0,1)$, there exists a sequence of approximated initial data $\left({\overrightarrow{u_{h}}}^{0},{\overrightarrow{u_{h}}}^{1}\right)$ such that

$$
\lim _{h \rightarrow 0} J_{h}\left({\overrightarrow{u_{h}}}^{0},{\overrightarrow{u_{h}}}^{1}\right)=J\left(u^{0}, u^{1}\right) .
$$


This is easy to see. Indeed, it is sufficient to take as $\left({\overrightarrow{u_{h}}}^{0},{\overrightarrow{u_{h}}}^{1}\right)$ the discrete initial data with same Fourier coefficients as $\left(u^{0}, u^{1}\right) \in \mathcal{D}$, which makes sense when $h$ is sufficiently small.

Finally, taking the hypotheses of the Theorem into account, and the strong convergence of the controls it is easy to see that the solutions $\vec{u}_{h}$ of (7) converge strongly to the solution $u$ of (1). Consequently convergences (198) and (199) are true.

Theorem 4.4. Let $T>0$. For any $h \in(0,1)$, consider the initial data $\left(\vec{y}_{h}^{0}, \vec{y}_{h}^{1}\right) \in \mathcal{H}_{h}^{1} \times \mathcal{H}_{h}^{-1} \quad$ and $\left(y^{0}, y^{1}\right) \in H_{0}^{1}(0,1) \times H^{-1}(0,1)$ of problems (64) and (6) as in (151).

Assume that,

$$
\begin{aligned}
& \left\{a_{k, h}^{0}\right\}_{k \in \mathbb{N}} \underset{h \rightarrow 0}{\longrightarrow}\left\{a_{k}^{0}\right\}_{k \in \mathbb{N}} \text { strongly in } \mathcal{H}^{1} \\
& \left\{b_{k, h}^{1}\right\}_{k \in \mathbb{N}} \underset{h \rightarrow 0}{\longrightarrow}\left\{b_{k}^{1}\right\}_{k \in \mathbb{N}} \text { strongly in } \mathcal{H}^{-1}
\end{aligned}
$$

Let $\vec{y}_{h}$ be the solution of system (64) with control $\nu_{h}$, so that both satisfy the decomposition given in (158). Then,

$$
\begin{aligned}
& \left\{c_{k, h}\right\}_{k \in \mathbb{N}} \underset{h \rightarrow 0}{\longrightarrow}\left\{c_{k}\right\}_{k \in \mathbb{N}} \text { strongly in } L^{\infty}\left(0, T ; \mathcal{H}^{1}\right) \cap W^{1, \infty}\left(0, T ; \mathcal{H}^{-1}\right), \\
& h\left\{e_{k, h}\right\}_{k \in \mathbb{N}} \underset{h \rightarrow 0}{\longrightarrow} 0 \quad \text { strongly in } \quad L^{\infty}\left(0, T ; \mathcal{H}^{-1}\right) \cap W^{1, \infty}\left(0, T ; \mathcal{H}^{-3}\right), \\
& h\left\{e_{k, h}\right\}_{k \in \mathbb{N}} \quad \text { is of order } h \text { in } \quad L^{\infty}\left(0, T ; \mathcal{H}^{-1}\right) \cap W^{1, \infty}\left(0, T ; \mathcal{H}^{-3}\right), \\
& \nu_{1, h}(.) \underset{h \rightarrow 0}{\longrightarrow} \nu(.) \quad \text { strongly in } L^{2}(0, T), \\
& h \nu_{2, h}^{\prime}(.) \underset{h \rightarrow 0}{\longrightarrow} 0 \quad \text { strongly in } H^{-1}(0, T), \\
& h \nu_{2, h}^{\prime}(.) \quad i s \text { of order } h \text { in } H^{-1}(0, T),
\end{aligned}
$$

where $y(x, t)=\sum_{k \in \mathbb{N}} c_{k}(t) \phi^{k}(x)$ is the solution of problem (6) with control $\nu(t)=-\partial_{x} u^{*}(1, t) \in L^{2}(0, T)$ where $u^{*}$ solves (1) with the initial data $\left(u^{0, *}, u^{1, *}\right)$ that minimizes the functional $J$ defined in (157).

Proof. The proof is similar to that of Theorem 4.3 and we omit it.

The authors are grateful to Sorin Micu for fruitful discussions.

\section{REFERENCES}

[1] J. Ball and M. Slemrod, Nonharmonic Fourier series and the stabilization of distributed semi-linear control systems. Comm. Pure Appl. Math. 37 (1979) 555-587.

[2] E. Crépeau, Exact Controllability of the Boussinesq Equation on a Bounded Domain. Adv. Differential Equations (to appear).

[3] A. Haraux, Séries lacunaires et contrôle semi-interne des vibrations d'une plaque rectangulaire. J. Math. Pures Appl. 68 (1989) 457-465.

[4] A.E. Ingham, Some trigonometrical inequalities with applications to the theory of series. Math. Z. 41 (1967) 367-379.

[5] J.A. Infante and E. Zuazua, Boundary observability for the space semi-discretizations of the 1-d wave equation. Math. Model. Numer. Anal. 33 (1999) 407-438.

[6] E. Isaacson and H.B. Keller, Analysis of numerical methods. John Wiley and Sons (1966).

[7] V. Komornik, Exact controllability and stabilization: The multiplier method. Masson and John Wiley, RAM 36 (1994).

[8] G. Lebeau, Contrôle de l' équation Schrödinger. J. Math. Pures Appl. 71 (1992) 267-291.

[9] L. León, Controle Exato da Equação da Viga 1-D Semi-discretizada no Espaço por Diferenças Finitas, Ph.D. Thesis. Instituto de Matemática, Universidade Federal de Rio de Janeiro (2001).

[10] J.L. Lions, Contrôlabilité exacte, stabilisation et perturbations de systèmes distribués, Tome 1. Masson, RMA 8, Paris (1988).

[11] J.L. Lions and E. Magenes, Problèmes aux limites non homogènes et applications, Vols. 1 and 2. Dunod, Paris (1968).

[12] S. Micu, Uniform Boundary Controllability of a Semi-Discrete 1-D Wave Equation. Numer. Math. (to appear). 
[13] S. Micu and E. Zuazua, Boundary controllability of a linear hybrid system arising in the control of noise. SIAM J. Control Optim. 35 (1997) 1614-1637.

[14] J. Simon, Compact sets in the space $L^{P}(0, T, B)$. Ann. Mat. Pura Appl. CXLVI (1987) 65-96.

[15] J.C. Strikwerda, Finite difference schemes and partial differential equation. Chapman and Hall (1995).

[16] J.W. Thomas, Numerical partial differential equations; finite difference methods. Springer, Texts Appl. Math. 22 (1995).

[17] R.M. Young, An introduction to nonharmonic Fourier series. Academic Press, Pure Appl. Math. A Series of Monographs and Textbooks (1980).

[18] E. Zuazua, Boundary observability for the finite space semi-discretization of the 2-d wave equation in the square. J. Math. Pures Appl. 78 (1999) 523-563.

[19] E. Zuazua, Contrôlabilité exacte en un temps arbitrairement petit de quelques modèles de plaques. Appendix I in [10] (1988) 465-491. 